\title{
The truncated Wigner method for Bose condensed gases: limits of validity and applications
}

\author{
Alice Sinatra, Carlos Lobo, and Yvan Castin \\ Laboratoire Kastler Brossel, Ecole Normale Supérieure, \\ UPMC and CNRS, 24 rue Lhomond, 75231 Paris Cedex 05, France
}

\begin{abstract}
We study the truncated Wigner method applied to a weakly interacting spinless Bose condensed gas which is perturbed away from thermal equilibrium by a time-dependent external potential. The principle of the method is to generate an ensemble of classical fields $\psi(r)$ which samples the Wigner quasi-distribution function of the initial thermal equilibrium density operator of the gas, and then to evolve each classical field with the Gross-Pitaevskii equation. In the first part of the paper we improve the sampling technique over our previous work [Jour. of Mod. Opt. 47, 2629$2644(2000)$ ] and we test its accuracy against the exactly solvable model of the ideal Bose gas. In the second part of the paper we investigate the conditions of validity of the truncated Wigner method. For short evolution times it is known that the time-dependent Bogoliubov approximation is valid for almost pure condensates. The requirement that the truncated Wigner method reproduces the Bogoliubov prediction leads to the constraint that the number of field modes in the Wigner simulation must be smaller than the number of particles in the gas. For longer evolution times the nonlinear dynamics of the noncondensed modes of the field plays an important role. To demonstrate this we analyse the case of a three dimensional spatially homogeneous Bose condensed gas and we test the ability of the truncated Wigner method to correctly reproduce the Beliaev-Landau damping of an excitation of the condensate. We have identified the mechanism which limits the validity of the truncated Wigner method: the initial ensemble of classical fields, driven by the time-dependent Gross-Pitaevskii equation, thermalises to a classical field distribution at a temperature $T_{\text {class }}$ which is larger than the initial temperature $T$ of the quantum gas. When $T_{\text {class }}$ significantly exceeds $T$ a spurious damping is observed in the Wigner simulation. This leads to the second validity condition for the truncated Wigner method, $T_{\text {class }}-T \ll T$, which requires that the maximum energy $\epsilon_{\max }$ of the Bogoliubov modes in the simulation does not exceed a few $k_{B} T$.
\end{abstract}

PACS numbers: 03.75.Fi, 05.10.Gg, 42.50.-p

\section{INTRODUCTION}

In Ref. [1] the formalism of the Wigner representation of the density operator, widely used in quantum optics, was proposed as a possible way to study the time evolution of Bose-Einstein condensates in the truncated Wigner approximation [2]. Like other existing approximate methods, such as the time-dependent Bogoliubov approach, it allows us to go beyond the commonly used Gross-Pitaevskii equation, in which the interactions between the condensate and the noncondensed atoms are neglected. Our aim in this paper is to illustrate the advantages and the limits of the truncated Wigner approach.

For reasons of clarity we will address two different situations in two separate parts of the paper: (i) the case of a stationary Bose condensed gas in thermal equilibrium and (ii) a time-dependent case when the gas is brought out of equilibrium by a known external perturbation. Even if the stationary gas is the starting point for both situations, the problems raised by the application of the Wigner method are of a different nature in the two cases.

(i) In the case of a Bose condensed gas in thermal equilibrium, the first step is to calculate the Wigner quasidistribution function associated with the $N$-body density operator $\hat{\sigma}$, which is a functional of a complex classical field $\psi(r)$. We showed in [3] that this is possible in the Bogoliubov approximation when the noncondensed fraction of atoms is small. With such an approximation, the Hamiltonian of the system is quadratic in the noncondensed field and its Wigner functional is a Gaussian. After that, we went through some more technical work to calculate the Wigner functional of the whole matter field including the condensate mode. In our derivation we made further approximations in addition to the Bogoliubov approximation. This introduces some artifacts in the Wigner functional as far as the condensate mode is concerned [3]. These artifacts are, however, insignificant when the number of thermally populated modes is much larger than one, or $k_{B} T \gg \hbar \omega$ in an isotropic trap of harmonic frequency $\omega$, so that the fluctuations in the number of condensate particles, due to finite temperature, are much larger than one. Once the Wigner functional for the Bose condensed gas in thermal equilibrium is calculated, the goal is to be able to sample it numerically in order to compute averages of observables and probability distributions. In practice, this step consists in generating a set of random atomic fields $\{\psi(r)\}$ according to a probability distribution dictated by the Wigner functional. We have now developed a more efficient algorithm to sample the Wigner functional in the case of spatially inhomogeneous condensates in a trapping potential than the one that we had presented in a previous paper [4], which 
we will explain here in detail. As far as the equilibrium Bose condensed gases are concerned, our method in its regime of validity, is equivalent to the $U(1)$ symmetry-preserving Bogoliubov approach of [5, 6], up to second order in the small parameter of the theory, which is the square root of the noncondensed fraction. Compared with the traditional Bogoliubov approach, our method presents, however, the practical advantage of avoiding the direct diagonalisation of the Bogoliubov matrix, which is a heavy numerical task in 2D and 3D in the absence of rotational symmetry. Moreover, due to the stochastic formulation we adopt, our method gives us access to single realisations and to the probability distribution of some observables such as the number of condensate particles, not easily accessible by the traditional Bogoliubov method. We show some examples where we compare the probability distribution of the number of condensate particles obtained with our method with an exact calculation in case of the ideal Bose gas.

(ii) Let us now consider the situation of a Bose condensed gas at thermal equilibrium which is brought out of equilibrium by a perturbation. The initial Wigner functional then evolves in time according to a kind of FokkerPlanck equation containing first and third order derivatives with respect to the atomic field. Numerical simulation of the exact evolution equation for the Wigner functional has intrinsic difficulties, as one would expect, since it represents the exact solution of the quantum many-body problem [7]. We are less ambitious here, and we rely on an approximation that consists in neglecting the third order derivatives in the evolution equation. This is known as the truncated Wigner approximation [1]. For a delta interaction potential between a finite number of low energy modes of the atomic field, the third order derivatives are expected to give a contribution which is smaller than that of the first order derivatives when the occupation numbers of the modes are much larger than unity. If we reason in terms of the stochastic fields $\psi(r, t)$ which sample the Wigner distribution at time $t$, then the truncated Wigner approximation corresponds to evolving the initial set of stochastic fields according to the Gross-Pitaevskii equation [8]:

$$
i \hbar \partial_{t} \psi=\left[-\frac{\hbar^{2}}{2 m} \Delta+U(r, t)+g|\psi|^{2}\right] \psi
$$

where $r$ is the set of single particle spatial coordinates, $m$ is the atom mass, $U$ is the trapping potential and $g$ is the coupling constant originating from the effective low energy interaction potential $V\left(r_{1}-r_{2}\right)=g \delta\left(r_{1}-r_{2}\right)$ and proportional to the $s$-wave scattering length $a$ of the true interaction potential, $g=4 \pi \hbar^{2} a / m$. Here, the crucial difference with respect to the usual Gross-Pitaevskii equation is that the field is now the whole matter field rather than the condensate field.

This procedure of evolving a set of random fields with the Gross-Pitaevskii equation is known as the classical field approximation, since equation (11) can be formally obtained from the Heisenberg equation of motion for the atomic field operator $\hat{\psi}$ by replacing the field operator by a c-number field. The classical field approximation has already been used in the Glauber-P representation to study the formation of the condensate [9, 10, 11, 12, 13. We face here a different situation: we assume an initially existing condensate and we use the Wigner representation, rather than the Glauber-P representation. The Wigner representation is in fact known in quantum optics to make the classical field approximation more accurate than in the Glauber-P representation because the "right amount" of quantum noise is contained in the initial state [14]. For a single mode system with a Kerr type nonlinearity and an occupation number $n$, the term neglected in the Wigner evolution equation is a third order derivative which is $1 / n^{2}$ times smaller than the classical field term, whereas the term neglected in the Glauber-P evolution equation is a second order derivative, which is only $1 / n$ times smaller than the classical field term. In the case of Bose-Einstein condensates however, we face a highly multimode problem and, therefore, the accuracy of the truncated Wigner approach needs to be revisited. We approach this problem in the second part of the paper. The strategy we adopt is to compare the predictions of the truncated Wigner method with existing well-established results: first with the time-dependent Bogoliubov approach and then with the Landau-Beliaev damping of a collective excitation in a spatially homogeneous condensate.

\section{BASIC NOTATIONS AND ASSUMPTIONS}

\section{A. Model Hamiltonian on a discrete grid}

Let us express a simple quantity like the mean atomic density using the Wigner representation:

$$
\left\langle\hat{\psi}^{\dagger}(r) \hat{\psi}(r)\right\rangle=\left\langle\psi^{*}(r) \psi(r)\right\rangle_{W}-\frac{1}{2}\left\langle\left[\hat{\psi}(r), \hat{\psi}^{\dagger}(r)\right]\right\rangle,
$$

where $\langle\ldots\rangle_{W}$ represents the average over the Wigner quasi-distribution function. This shows that the discretisation of the problem on a finite grid is necessary to avoid infinities: in the continuous version of the problem, $\left[\hat{\psi}(r), \hat{\psi}^{\dagger}(r)\right]=$ $\delta(0)=+\infty$. Physically this divergence comes from the fact that, in the Wigner point of view, some noise is included in each mode of the classical field $\psi$ to mimic quantum noise; this extra noise adds up to infinity for a system with 
an infinite number of modes. Therefore we use, from the beginning, a discrete formulation of our problem which will make it also suitable for numerical simulations.

We consider a discrete spatial grid forming a box of length $L_{\nu}$ along the direction $\nu=x, y, z$ with an even number $n_{\nu}$ of equally spaced points. We denote $\mathcal{N} \equiv \prod_{\nu} n_{\nu}$ the number of points on the grid, $V \equiv \prod_{\nu} L_{\nu}$ the volume of the grid and $d V \equiv V / \mathcal{N}$ the volume of the unit cell of the grid. We take periodic boundary conditions in the box [15]. We can then expand the field operator over plane waves

$$
\hat{\psi}(r)=\sum_{k} \hat{a}_{k} \frac{1}{\sqrt{V}} e^{i k \cdot r}
$$

where $\hat{a}_{k}$ annihilates a particle of momentum $k$ and where the components of $k$ are $k_{\nu}=2 \pi j_{\nu} / L_{\nu}$ with the integers $j_{\nu}$ running from $-n_{\nu} / 2$ to $n_{\nu} / 2-1$. We then have the inverse formula:

$$
\hat{a}_{k}=d V \sum_{r} \frac{1}{\sqrt{V}} e^{-i k \cdot r} \hat{\psi}(r)
$$

For each node $r_{i}$ on the spatial grid, we find the commutation relations for the field operator:

$$
\left[\hat{\psi}\left(r_{i}\right), \hat{\psi}^{\dagger}\left(r_{j}\right)\right]=\frac{1}{d V} \delta_{i, j}
$$

and the discretised model Hamiltonian that we use is:

$$
\hat{H}=\sum_{k} \frac{\hbar^{2} k^{2}}{2 m} \hat{a}_{k}^{\dagger} \hat{a}_{k}+d V \sum_{r} U(r) \hat{\psi}^{\dagger}(r) \hat{\psi}(r)+\frac{g}{2} d V \sum_{r} \hat{\psi}^{\dagger}(r) \hat{\psi}^{\dagger}(r) \hat{\psi}(r) \hat{\psi}(r) .
$$

The first term in (6) is the kinetic energy, which is easy to calculate in the momentum representation. In the position representation, the kinetic energy is a matrix that couples the $\mathcal{N}$ points of the grid. In the following we will write it as $p^{2} / 2 m$. The second term is the trapping potential. The last term represents the atomic interactions modeled by a discrete Kronecker $\delta$ potential

$$
V\left(r_{1}-r_{2}\right)=\frac{g}{d V} \delta_{r_{1}, r_{2}}
$$

with a coupling constant $g=4 \pi \hbar^{2} a / m$, where $a$ is the $s$-wave scattering length of the true interaction potential.

We indicate briefly some requirements for the discrete Hamiltonian to be a good representation of reality. First, the spatial step of the grid should be smaller than the macroscopic physical scales of the problem:

$$
d x_{\nu} \ll \xi \text { and } d x_{\nu} \ll \lambda,
$$

where $\xi=1 / \sqrt{8 \pi \rho|a|}$ is the healing length for the maximal atomic density $\rho$ and $\lambda=\sqrt{2 \pi \hbar^{2} / m k_{B} T}$ is the thermal de Broglie wavelength at temperature $T$. Secondly, the spatial step of the grid should be larger than the absolute value of the scattering length $a$ :

$$
d x_{\nu} \gg|a|
$$

so that the scattering amplitude of the model potential (7) is indeed very close to $a$. Another way of saying this is that the model potential (7) can be treated in the Born approximation for the low energy waves. A more precise treatment, detailed in the appendix A, is to replace in (7) the coupling constant $g$ by its bare value $g_{0}$ adjusted so that the scattering length of the model potential on the grid is exactly equal to $a$.

\section{B. Wigner representation}

The Wigner quasi-distribution function associated with the $N$-body density operator $\hat{\sigma}$ is defined as the Fourier transform of the characteristic function $\chi$ :

$$
\begin{aligned}
W(\psi) & \equiv \int \prod_{r} \frac{d \operatorname{Re} \gamma(r) d \operatorname{Im} \gamma(r) d V}{\pi^{2}} \chi(\gamma) e^{d V \sum_{r} \gamma^{*}(r) \psi(r)-\gamma(r) \psi^{*}(r)} \\
\chi(\gamma) & =\operatorname{Tr}\left[\hat{\sigma} e^{d V \sum_{r} \gamma(r) \hat{\psi}^{\dagger}(r)-\gamma^{*}(r) \hat{\psi}(r)}\right]
\end{aligned}
$$


where $\gamma(r)$ is a complex field on the spatial grid and $\hat{\sigma}$ is the density operator of the system. With this definition the Wigner function is normalised to unity:

$$
\int \prod_{r} d \operatorname{Re} \psi(r) d \operatorname{Im} \psi(r) d V W(\psi)=1 .
$$

We recall that the moments of the Wigner function correspond to totally symmetrised quantum expectation values, i.e.,

$$
\left\langle O_{1} \ldots O_{n}\right\rangle_{W}=\frac{1}{n !} \sum_{P} \operatorname{Tr}\left[\hat{O}_{P(1)} \ldots \hat{O}_{P(n)} \hat{\sigma}\right]
$$

where the sum is taken over all the permutations $P$ of $n$ objects, $O_{k}$ stands for $\psi$ or $\psi^{*}$ in some point of the grid and $\hat{O}_{k}$ is the corresponding field operator.

The equation of motion for the density operator $\hat{\sigma}$

$$
\frac{d}{d t} \hat{\sigma}=\frac{1}{i \hbar}[\hat{H}, \hat{\sigma}]
$$

can be written exactly as the following equation of motion for the Wigner distribution:

$$
i \hbar \frac{\partial W}{\partial t}=\sum_{r} \frac{\partial}{\partial \psi(r)}\left(-f_{\psi} W\right)+\frac{g}{4(d V)^{2}} \frac{\partial^{3}}{\partial^{2} \psi(r) \partial \psi^{*}(r)}(\psi(r) W)-\text { c.c. },
$$

with a drift term

$$
f_{\psi}=\left[\frac{p^{2}}{2 m}+U(r, t)+g \psi^{*} \psi-\frac{g}{d V}\right] \psi .
$$

The truncated Wigner approximation consists in neglecting the cubic derivatives in the equation for $W$. The resulting equation reduces to the drift term whose effect amounts to evolving the field $\psi$ according to an equation which resembles the Gross-Pitaevskii equation (11). The constant term $-g / d V$ inside the brackets of the above equation can be eliminated by a redefinition of the global phase of $\psi$, which has no physical consequence for observables conserving the number of particles.

\section{SAMPLING THE WIGNER FUNCTIONAL FOR A BOSE CONDENSED GAS IN THERMAL EQUILIBRIUM}

In [3] we derive an expression of the Wigner functional for a Bose condensed gas in thermal equilibrium in the frame of the $U(1)$ symmetry-preserving Bogoliubov approach [5, [6], in which the gas has a fixed total number of particles equal to $N$. We first introduce the approximate condensate wavefunction $\phi(r)$, which is a solution of the time-independent Gross-Pitaevskii equation:

$$
H_{\mathrm{gp}} \phi \equiv\left[\frac{p^{2}}{2 m}+U(r, t=0)+N g|\phi|^{2}-\mu\right] \phi=0 .
$$

We then split the classical field $\psi(r)$ into components orthogonal and parallel to the condensate wavefunction $\phi(r)$ :

$$
\begin{aligned}
\psi(r) & =a_{\phi} \phi(r)+\psi_{\perp}(r) \\
a_{\phi} & \equiv d V \sum_{r} \phi^{*}(r) \psi(r) .
\end{aligned}
$$

The Wigner functional provides us with the joint probability distributions of the transverse classical field $\psi_{\perp}(r)$, that we call the noncondensed field, and of the complex amplitude $a_{\phi}$. Due to the $U(1)$ symmetry-preserving character of the theory, the final Wigner functional is of the form [3]

$$
W(\psi)=\int \frac{d \theta}{2 \pi} W_{0}\left(e^{-i \theta} \psi\right) .
$$

This means that one can sample the distribution $W(\psi)$ by (i) choosing a random field $\psi$ according to the distribution $W_{0}(\psi)$, (ii) choosing a random global phase $\theta$ uniformly distributed between 0 and $2 \pi$, and (iii) forming the total atomic field as $\psi_{\text {tot }}(r)=e^{i \theta} \psi(r)$. In practice, the global phase factor $e^{i \theta}$ is unimportant to calculate the expectation value of observables that conserve the number of particles. Since the other observables have a vanishing mean value, we can limit ourselves to the sampling of the $\theta=0$ component of the Wigner functional, $W_{0}(\psi)$. 


\section{A. Sampling the distribution of the noncondensed field}

The first step of the sampling procedure consists in generating a set of noncondensed fields $\left\{\psi_{\perp}\right\}$ according to the probability distribution

$$
P\left(\psi_{\perp}\right) \propto \exp \left[-d V\left(\psi_{\perp}^{*}, \psi_{\perp}\right) \cdot M\left(\begin{array}{c}
\psi_{\perp} \\
\psi_{\perp}^{*}
\end{array}\right)\right]
$$

where we have collected all the components of $\psi_{\perp}$ and $\psi_{\perp}^{*}$ in a single vector with $2 \mathcal{N}$ components, $M$ is the $2 \mathcal{N} \times 2 \mathcal{N}$ matrix:

$$
M=\eta \tanh \frac{\mathcal{L}}{2 k_{B} T}
$$

with

$$
\eta=\left(\begin{array}{rr}
1 & 0 \\
0 & -1
\end{array}\right)
$$

and where $\mathcal{L}$ is a $2 \mathcal{N} \times 2 \mathcal{N}$ matrix, which is the discretised version of the Bogoliubov operator of [5]:

$$
\mathcal{L}=\left(\begin{array}{cc}
H_{\mathrm{gp}}+N g \mathcal{Q}|\phi|^{2} \mathcal{Q} & N g \mathcal{Q} \phi^{2} \mathcal{Q}^{*} \\
-N g \mathcal{Q}^{*} \phi^{* 2} \mathcal{Q} & -H_{\mathrm{gp}}^{*}-N g \mathcal{Q}^{*}|\phi|^{2} \mathcal{Q}^{*}
\end{array}\right)
$$

In this expression the $\mathcal{N} \times \mathcal{N}$ matrix $\mathcal{Q}$ projects orthogonally to the condensate wavefunction $\phi$ in the discrete spatial grid $\left\{r_{i}\right\}$ representation,

$$
\mathcal{Q}_{i j}=\delta_{i j}-d V \phi\left(r_{i}\right) \phi^{*}\left(r_{j}\right)
$$

Note that the matrix $M$ can be shown to be Hermitian from the fact that $\mathcal{L}^{\dagger}=\eta \mathcal{L} \eta$.

\section{Direct diagonalisation of $\mathcal{L}$}

If the eigenvectors of $\mathcal{L}$ are known, we can use the following modal expansion:

$$
\left(\begin{array}{c}
\psi_{\perp} \\
\psi_{\perp}^{*}
\end{array}\right)=\sum_{k} b_{k}\left(\begin{array}{c}
u_{k} \\
v_{k}
\end{array}\right)+b_{k}^{*}\left(\begin{array}{c}
v_{k}^{*} \\
u_{k}^{*}
\end{array}\right)
$$

where the sum is to be taken over all eigenmodes $\left(u_{k}, v_{k}\right)$ of $\mathcal{L}$ normalisable as $\left\langle u_{k} \mid u_{k}\right\rangle-\left\langle v_{k} \mid v_{k}\right\rangle=1$, with corresponding eigenvalues $\epsilon_{k}$. Since the condensate is assumed to be in a thermodynamically stable or metastable state, all the $\epsilon_{k}$ are positive [16]. The probability distribution (21) is then a simple product of Gaussian distributions for the complex amplitudes $b_{k}$ :

$$
P_{k}\left(b_{k}\right)=\frac{2}{\pi} \tanh \left(\frac{\epsilon_{k}}{2 k_{B} T}\right) \exp \left[-2\left|b_{k}\right|^{2} \tanh \left(\frac{\epsilon_{k}}{2 k_{B} T}\right)\right] .
$$

Each Gaussian distribution is easily sampled numerically [17]. Note that, even at zero temperature, the Gaussian distribution has a nonzero width: this is a signature of the extra noise introduced in the Wigner representation to mimic quantum noise.

\section{Brownian motion simulation}

The sampling of the distribution (21) can actually be performed without diagonalisation of $\mathcal{L}$ (an advantage for spatially inhomogeneous Bose condensates in the absence of rotational symmetry [4]) by means of a Brownian motion simulation for the noncondensed field:

$$
d\left(\begin{array}{c}
\psi_{\perp} \\
\psi_{\perp}^{*}
\end{array}\right)=-\alpha d t\left(\begin{array}{c}
\psi_{\perp} \\
\psi_{\perp}^{*}
\end{array}\right)+Y\left(\begin{array}{c}
d \xi \\
d \xi^{*}
\end{array}\right)
$$


where the field $d \xi$ is the noise term. The time $t$ here is a purely fictitious time with no physical meaning and will be taken to be dimensionless. On our discrete grid, $\psi_{\perp}$ is a vector with $\mathcal{N}$ components, $d \xi$ is a Gaussian random vector of $\mathcal{N}$ components with zero mean and a covariance matrix $\left\langle d \xi_{i} d \xi_{j}^{*}\right\rangle$ equal to $(2 d t / d V) \delta_{i, j}$, while $\alpha, Y$ are $2 \mathcal{N} \times 2 \mathcal{N}$ matrices. To ensure that the Brownian motion relaxes towards the correct probability distribution (21) we require that the drift matrix $\alpha$ and the diffusion matrix $D \equiv Y\left(Y^{\dagger}\right)$ satisfy a generalised Einstein's relation [4]:

$$
D^{-1} \alpha=\alpha^{\dagger} D^{-1}=2 M,
$$

where $M$ is the matrix (22). There is, of course, no unique choice for $\alpha$ and $Y$. With respect to our previous work [4], we have largely improved the efficiency of our simulation by a different choice of $\alpha, Y$ and by the use of a second order integration scheme of the stochastic differential equation (28), more efficient than the usual first order Euler's scheme. In the appendix $B$ we give a detailed description of these improvements, useful to the reader who is interested in implementing the numerical algorithm.

\section{B. Sampling the condensate amplitude}

We now have to sample the condensate amplitude $a_{\phi}$ from the Wigner functional $W_{0}$. This amplitude turns out to be real, and can be written as

$$
a_{\phi}=\sqrt{N_{0}} \quad \text { where } \quad N_{0}=a_{\phi}^{*} a_{\phi} .
$$

Since we already know how to generate the noncondensed part of the field $\psi_{\perp}$, we have to sample the conditional distribution $P\left(N_{0} \mid \psi_{\perp}\right)$.

Due to a first approximation that we have performed in [3], which consists in treating "classically" the condensate mode and neglecting its quantum fluctuations in the limit of a large number of condensate particles, the probability distribution $P\left(N_{0}\right)$, that we will obtain by averaging $P\left(N_{0} \mid \psi_{\perp}\right)$ over the stochastic realisations of the noncondensed field $\psi_{\perp}$, actually coincides with the probability distribution of the number of condensed particles $\hat{a}_{\phi}^{\dagger} \hat{a}_{\phi}$ so that within this approximation we have:

$$
\begin{aligned}
\left\langle N_{0}\right\rangle & =\left\langle\hat{a}_{\phi}^{\dagger} \hat{a}_{\phi}\right\rangle, \\
\operatorname{Var}\left(N_{0}\right) & =\operatorname{Var}\left(\hat{a}_{\phi}^{\dagger} \hat{a}_{\phi}\right), \ldots
\end{aligned}
$$

Note that this should not be the case for the exact Wigner distribution as, e.g., the average $\left\langle N_{0}\right\rangle$ should be equal to $\left\langle\hat{a}_{\phi}^{\dagger} \hat{a}_{\phi}\right\rangle+1 / 2$ and the variance of $N_{0}$ should exceed the variance of $\hat{a}_{\phi}^{\dagger} \hat{a}_{\phi}$ by $1 / 4$.

We show in [3] that, when the number of thermally populated modes is much larger than one, the width in $N_{0}$ of the conditional distribution $P\left(N_{0} \mid \psi_{\perp}\right)$ is much narrower than the width of the distribution $P\left(N_{0}\right)$, so that we can replace the distribution $P\left(N_{0} \mid \psi_{\perp}\right)$ by a delta function centered on its mean value. With this second, more severe, approximation we get for the sampling:

$$
N_{0} \simeq \operatorname{Mean}\left(N_{0} \mid \psi_{\perp}\right)=C-\frac{1}{2} d V\left(\psi_{\perp}^{*}, \psi_{\perp}\right) \cdot\left[\operatorname{Id}-M^{2}\right]\left(\begin{array}{c}
\psi_{\perp} \\
\psi_{\perp}^{*}
\end{array}\right)
$$

where the constant $C$ is finite only in the discretised version and is given by

$$
C=N-\frac{1}{4} \operatorname{Tr} M+\frac{1}{2} \operatorname{Tr} \mathcal{Q} .
$$

Here, the trace of the projector $\mathcal{Q}$ is simply the number of modes in the simulation minus one.

The second approximation (33) does not introduce errors in the average $\left\langle N_{0}\right\rangle$. We are able to verify a posteriori that the error introduced in the variance $\left\langle N_{0}^{2}\right\rangle-\left\langle N_{0}\right\rangle^{2}$ is small in the following way: on one hand we calculate the variance of $N_{0}\left(\operatorname{Var}\left(N_{0}\right)\right)$, by using (33). On the other hand we calculate the variance $\operatorname{Var}(\delta \hat{N})$ of the number of noncondensed particles by using directly the ensemble of noncondensed fields $\left\{\psi_{\perp}\right\}$. Since the total number of particles is fixed one should have $\operatorname{Var}\left(N_{0}\right)=\operatorname{Var}\left(\hat{a}_{\phi}^{\dagger} \hat{a}_{\phi}\right)=\operatorname{Var}\left(\hat{\psi}_{\perp}^{\dagger} \hat{\psi}_{\perp}\right)$, and deviation from this identity gives us the error of $\operatorname{Var}\left(N_{0}\right)$.

We are now ready to form the total field:

$$
\psi(r)=\sqrt{N_{0}}\left(\phi(r)+\frac{\phi_{\perp}^{(2)}(r)}{N}\right)+\psi_{\perp}(r) .
$$

The function $\phi_{\perp}^{(2)}$ is a correction to the condensate wavefunction including the condensate depletion neglected in the Gross-Pitaevskii equation (17) and the mean field effect of the noncondensed particles. This correction can be calculated from the ensemble of noncondensed fields $\left\{\psi_{\perp}\right\}$ as explained in [4]. As we will see in section IVA] its contribution to the one-body density matrix is of the same order as that of $\psi_{\perp}$ and therefore has to be included. 


\section{Tests and applications: Distribution of the number of condensate particles}

We can use the sampling procedure described above to calculate some equilibrium properties of the Bose condensed gas. Recently, the variance of the number of particles in the condensate has drawn increasing attention [18, 19, 20]. In our case we have access to the whole probability distribution for $N_{0}$ by applying equation (33) to the ensemble of stochastic noncondensed fields $\left\{\psi_{\perp}\right\}$.

\section{Ideal Bose gas}

As a test we check our probability distribution for the number of condensate particles against the exact one for the ideal Bose gas $(g=0)$ in one and two dimensions. The results are in figure 1 .
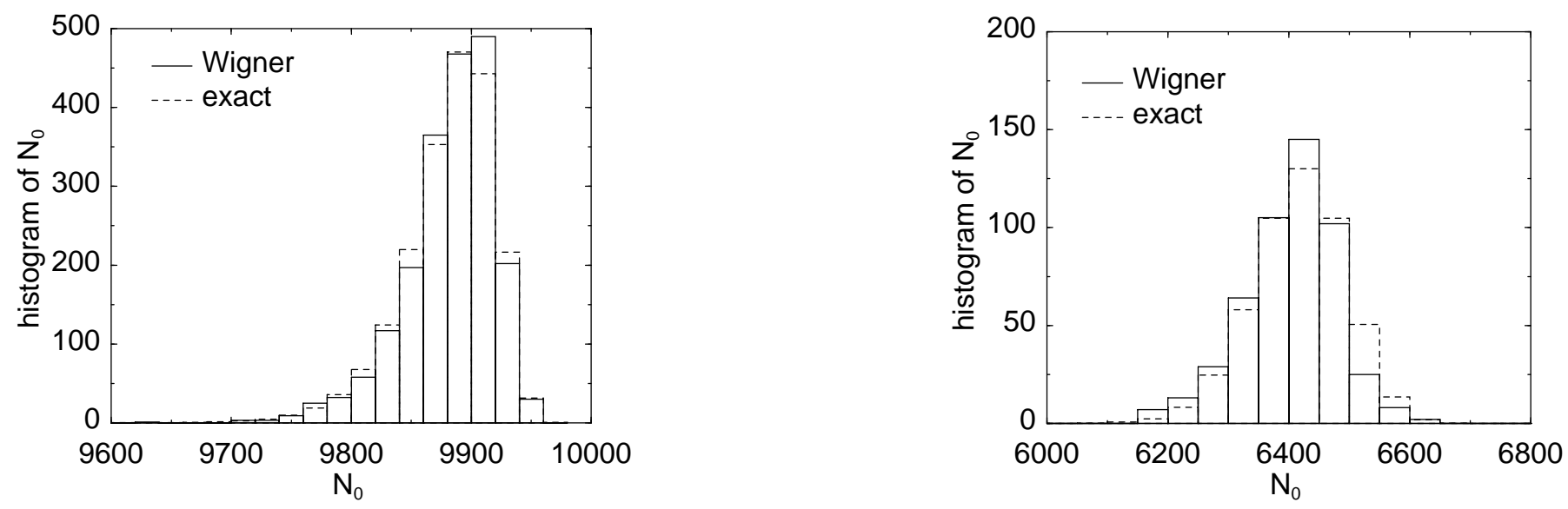

FIG. 1: Probability distribution in the canonical ensemble of the number of condensate particles for the ideal Bose gas in thermal equilibrium in an isotropic harmonic potential $U(r)=\frac{1}{2} m \omega^{2} r^{2}$. (a) In a $1 \mathrm{D}$ model for $k_{B} T=30 \hbar \omega$, and $N=10000$. For the Wigner simulation 2000 realisations have been performed on a grid with 128 points. For the exact Bogoliubov rejection method described in the end of this subsection on the ideal gas, 400000 realisations have been performed so that the statistical error is less than one per cent for the most populated channels of the histogram. (b) In a $2 \mathrm{D}$ model for $k_{B} T=30 \hbar \omega$, and $N=8000$. For the Wigner simulation 500 realisations have been performed on a grid with $128 \times 128$ points. For the exact sampling 100000 realisations have been performed.

The distributions of the number of condensed particles $N_{0}$ are clearly not Gaussian. To characterise them, besides the mean and the variance of $N_{0}$ one can introduce the skewness defined as:

$$
\operatorname{skew}\left(N_{0}\right)=\frac{\left\langle\left(N_{0}-\left\langle N_{0}\right\rangle\right)^{3}\right\rangle}{\left(\left\langle N_{0}^{2}\right\rangle-\left\langle N_{0}\right\rangle^{2}\right)^{3 / 2}} .
$$

For the parameters of figure 1 we give the mean, the standard deviation and the skewness of $N_{0}$ obtained from the simulation, together with their exact values:

\begin{tabular}{|c||c|c||c|c|}
\hline & 1D simulation & 1D exact & 2D simulation & $2 \mathrm{D}$ exact \\
\hline$\left\langle N_{0}\right\rangle$ & 9882. & 9880. & 6403. & 6415. \\
\hline$\Delta N_{0}$ & 37.5 & 38.3 & 75.9 & 77.1 \\
\hline skew $\left(N_{0}\right)$ & -1.20 & -1.16 & -0.40 & -0.334 \\
\hline
\end{tabular}

In what follows we explain in some detail how the exact probability distribution for the ideal Bose gas is obtained. Let $\hat{\sigma}$ be the density operator for the ideal Bose gas in the canonical ensemble:

$$
\hat{\sigma}=\frac{1}{Z} e^{-\beta \hat{H}} p_{N}
$$


The operator $p_{N}$ projects onto the subspace with $N$ particles, and $\hat{H}=\sum_{k} \epsilon_{k} \hat{a}_{k}^{\dagger} \hat{a}_{k}$ is written in the eigenbasis of the trapping potential. In the spirit of the number conserving Bogoliubov method, we eliminate the condensate mode by writing

$$
\hat{a}_{0}^{\dagger} \hat{a}_{0}=\hat{N}-\sum_{k \neq 0} \hat{a}_{k}^{\dagger} \hat{a}_{k}
$$

Since the total number of particles is fixed we can replace the operator $\hat{N}$ by the c-number $N$ in (38). Furthermore we establish a one to one correspondence between (i) each configuration of excited modes $\left\{n_{k}, k>0\right\}$ having a number of excited particles $N^{\prime}=\sum_{k} n_{k}$ lower than $N$ and (ii) each configuration of the whole system with $n_{k}$ particles in excited mode $k$ and $N-N^{\prime}$ particles in the condensate. We then obviously have to reject the configurations of excited modes for which the number of particles in the excited states $N^{\prime}$ is larger than $N$. This amounts to reformulating the effect of the projector $p_{N}$ in terms of an Heaviside function $Y$. We then rewrite $\hat{\sigma}$ as:

$$
\hat{\sigma}=\frac{1}{Z} e^{-\beta \epsilon_{0} N} e^{-\beta \sum_{k \neq 0}\left(\epsilon_{k}-\epsilon_{0}\right) \hat{a}_{k}^{\dagger} \hat{a}_{k}} Y\left(N-\sum_{k \neq 0} \hat{a}_{k}^{\dagger} \hat{a}_{k}\right) .
$$

For the sampling procedure we use a rejection method i.e. we sample the probability distribution of the number of particles $n_{k}$ in each mode $k \neq 0$ without the constraint imposed by the Heaviside function and we reject configurations with $N^{\prime}>N$. In this scheme we have to generate the $n_{k}, k=1, \ldots, \mathcal{N}$, according to the probability distribution

$$
p_{k}\left(n_{k}\right)=\lambda_{k}^{n_{k}}\left(1-\lambda_{k}\right) \quad \text { with } \quad \lambda_{k}=e^{-\beta\left(\epsilon_{k}-\epsilon_{0}\right)}
$$

For each $k$ we proceed as follows: in a loop over $n_{k}$ starting from 0 we generate a random number $\epsilon$ uniformly distributed in the interval $[0,1]$ and we compare it with $\lambda_{k}$ : if $\epsilon<\lambda_{k}$, we proceed with the next step of the loop, otherwise we exit from the loop and the current value of $n_{k}$ is returned.

The calculation can also be done in the Bogoliubov approximation, that is by neglecting the Heaviside function in (39). For the parameters of figure 1 this is actually an excellent approximation, as the mean population of the condensate mode is much larger than its standard deviation, and the corresponding approximate results are in practice indistinguishable from the exact ones. The predictions of this Bogoliubov approximation for the first three moments of $N_{0}$ involve a sum over all the excited modes of the trapping potential:

$$
\begin{aligned}
\left\langle N_{0}\right\rangle & =N-\sum_{k \neq 0} \bar{n}_{k} \\
\operatorname{Var}\left(N_{0}\right) & =\sum_{k \neq 0} \bar{n}_{k}\left(1+\bar{n}_{k}\right) \\
\left\langle\left(N_{0}-\left\langle N_{0}\right\rangle\right)^{3}\right\rangle & =\sum_{k \neq 0} 2 \bar{n}_{k}^{3}+3 \bar{n}_{k}^{2}+\bar{n}_{k}
\end{aligned}
$$

where $\bar{n}_{k}=1 /\left(\exp \left(\beta\left(\epsilon_{k}-\epsilon_{0}\right)\right)-1\right)$ is the mean occupation number of the mode $k$. In the limit $k_{B} T \gg \hbar \omega$ for an isotropic harmonic trap an analytical calculation, detailed in the appendix $\mathrm{C}$. shows that the skewness tends to a constant in $1 \mathrm{D}$, tends to zero logarithmically in $2 \mathrm{D}$ and tends to zero polynomially in $3 \mathrm{D}$ [21]:

$$
\begin{aligned}
& \operatorname{skew}_{1 \mathrm{D}}\left(N_{0}\right) \simeq-\frac{2 \zeta(3)}{\zeta(2)^{3 / 2}}=-1.139547 \ldots \\
& \operatorname{skew}_{2 \mathrm{D}}\left(N_{0}\right) \simeq-\frac{2(\zeta(2)+\zeta(3))}{\left(\log \left(k_{B} T / \hbar \omega\right)+1+\gamma+\zeta(2)\right)^{3 / 2}} \\
& \operatorname{skew}_{3 \mathrm{D}}\left(N_{0}\right) \simeq-\frac{\log \left(k_{B} T / \hbar \omega\right)+\gamma+\frac{3}{2}+3 \zeta(2)+2 \zeta(3)}{\left(k_{B} T / \hbar \omega\right)^{3 / 2}\left\{\zeta(2)+\left(3 \hbar \omega / 2 k_{B} T\right)\left[\log \left(k_{B} T / \hbar \omega\right)+\gamma+1-\zeta(2) / 3\right]\right\}^{3 / 2}}
\end{aligned}
$$

where $\zeta$ is the Riemann Zeta function and $\gamma=0.57721 \ldots$ is Euler's constant.

\section{Interacting case}

As an example we show in figure 2 the probability distribution for the number of condensate particles in the interacting case to demonstrate that the large skewness of $N_{0}$ in $1 \mathrm{D}$ can even be enhanced in presence of interaction: 
the skewness of $N_{0}$ in figure 2 is equal to -2.3 . We have been able 22] to calculate $P\left(N_{0}\right)$ in the Bogoliubov approximation in the interacting case starting from the sampling of the Wigner distribution of the noncondensed field (21). We compare the results with the Wigner approach in the same figure. As expected the agreement is excellent in the regime $k_{B} T=30 \hbar \omega \gg \hbar \omega$.

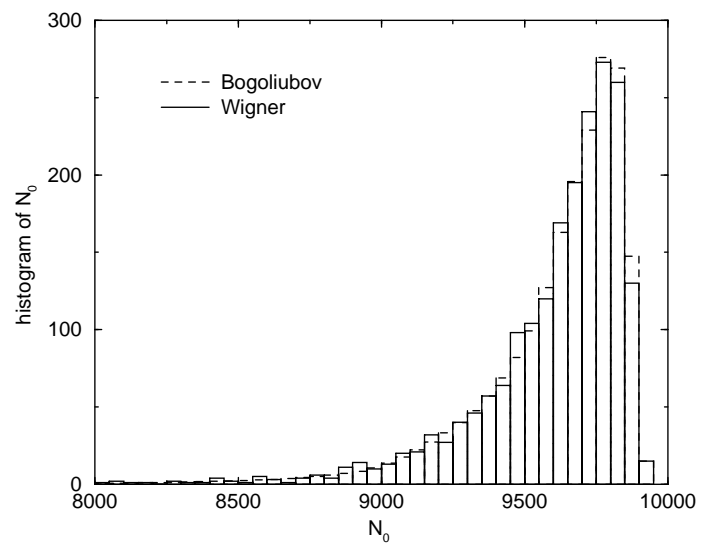

FIG. 2: Probability distribution of the number of condensate particles in the canonical ensemble for a 1D interacting Bose gas in thermal equilibrium in a harmonic trap $U(x)=\frac{1}{2} m \omega^{2} x^{2}$, with $k_{B} T=30 \hbar \omega, \mu=14.1 \hbar \omega$ and $N=10000$, corresponding to a coupling constant $g=0.01 \hbar \omega(\hbar / m \omega)^{1 / 2}$. The results have been obtained with the Wigner method using 2000 realisations on a grid with 128 points. The dashed line is the histogram of the probability distribution of $N_{0}$ in the Bogoliubov approximation generated using the same 2000 realisations, obtained with a method described in [22].

\section{THE TRUNCATED WIGNER METHOD FOR A TIME-DEPENDENT BOSE CONDENSED GAS}

In this section we investigate the conditions of validity of the truncated Wigner approach for time-dependent BoseEinstein condensates. The strategy that we adopt is to compare the predictions of the truncated Wigner approach to well-established theories: the time-dependent Bogoliubov approach in section IVA and the Landau-Beliaev damping of a collective excitation in a spatially homogeneous condensate, in section IVB

\section{A. The truncated Wigner method vs the time-dependent Bogoliubov method}

In this section we investigate analytically the equivalence between the time-dependent Bogoliubov approach of [5] and the truncated Wigner method in the limit in which the noncondensed fraction is small.

We begin by sketching the number conserving Bogoliubov method of Ref. [5]. We split the atomic field operator into components parallel and orthogonal to the exact time-dependent condensate wavefunction $\phi_{\text {ex }}$ [23] (omitting for simplicity the time label for the field operators and for the condensate wavefunction):

$$
\hat{\psi}(r)=\hat{a}_{\phi_{\mathrm{ex}}} \phi_{\mathrm{ex}}(r)+\hat{\psi}_{\perp}(r)
$$

and we consider the limit

$$
N \rightarrow \infty \quad N g=\text { constant } \quad T=\text { constant } \quad \mathcal{N}=\text { constant }
$$

In [5] one performs a formal systematic expansion in powers of $1 / \sqrt{N}$ of the exact condensate wavefunction $\phi_{\mathrm{ex}}$

$$
\phi_{\mathrm{ex}}(r)=\phi(r)+\frac{\phi^{(1)}(r)}{\sqrt{N}}+\frac{\phi^{(2)}(r)}{N}+\ldots
$$

and of the noncondensed field

$$
\hat{\Lambda}_{\mathrm{ex}}(r) \equiv \frac{1}{\sqrt{N}} \hat{a}_{\phi_{\mathrm{ex}}}^{\dagger} \psi_{\perp}(r)=\hat{\Lambda}(r)+\frac{1}{\sqrt{N}} \hat{\Lambda}^{(1)}(r)+\ldots
$$


Note that in the lowest order approximation to $\hat{\Lambda}_{\mathrm{ex}}$ the exact condensate wavefunction $\phi_{\mathrm{ex}}$ is replaced by the solution $\phi$ of the time-dependent Gross-Pitaevskii equation

$$
i \hbar \partial_{t} \phi=\left[p^{2} / 2 m+U(r, t)+N g|\phi|^{2}\right] \phi
$$

and $\hat{a}_{\phi} / \sqrt{N}$ is replaced by the phase operator $\hat{A}_{\phi}=\hat{a}_{\phi}\left(\hat{a}_{\phi}^{\dagger} \hat{a}_{\phi}\right)^{-1 / 2}$ so that

$$
\hat{\Lambda}(r)=\frac{1}{\sqrt{\hat{a}_{\phi}^{\dagger} \hat{a}_{\phi}}} \hat{a}_{\phi}^{\dagger}\left[\hat{\psi}(r)-\phi(r) \hat{a}_{\phi}\right]
$$

and $\hat{\Lambda}(r)$ satisfies bosonic commutation relations

$$
\left[\hat{\Lambda}(r), \hat{\Lambda}^{\dagger}(s)\right]=\frac{1}{d V} \mathcal{Q}_{r, s}
$$

where the matrix $\mathcal{Q}_{r, s}=\delta_{r, s}-d V \phi(r) \phi^{*}(s)$ projects orthogonally to $\phi$. To the first two leading orders in $1 / \sqrt{N}$ one obtains an approximate form of the one-body density matrix:

$$
\begin{aligned}
\langle r|\rho| s\rangle \equiv\left\langle\hat{\psi}^{\dagger}(s) \hat{\psi}(r)\right\rangle & =(N-\langle\delta \hat{N}\rangle) \phi(r) \phi^{*}(s) \\
& +\left\langle\hat{\Lambda}^{\dagger}(s) \hat{\Lambda}(r)\right\rangle \\
& +\phi^{*}(s) \phi_{\perp}^{(2)}(r)+\phi(r) \phi_{\perp}^{(2) *}(s) \\
& +O\left(\frac{1}{\sqrt{N}}\right) .
\end{aligned}
$$

We call the first term "parallel-parallel" because it originates from the product of two parts of the field both parallel to the condensate wavefunction; it describes the physics of a pure condensate with $N-\langle\delta \hat{N}\rangle$ particles. The second term, which we call "orthogonal-orthogonal" because $\hat{\Lambda}$ is orthogonal to $\phi$, describes the noncondensed particles in the Bogoliubov approximation. The third term, called "orthogonal-parallel", describes corrections to the Gross-Pitaevskii condensate wavefunction due to the presence of noncondensed particles [5]. In (50) $\langle\delta \hat{N}\rangle$ is the average number of noncondensed particles in the Bogoliubov approximation:

$$
\langle\delta \hat{N}\rangle=\sum_{r} d V\left\langle\hat{\Lambda}^{\dagger}(r) \hat{\Lambda}(r)\right\rangle
$$

The evolution equations for $\hat{\Lambda}$ and $\phi_{\perp}^{(2)}$ are given in appendix D]

Having described the Bogoliubov method, let us now consider the truncated Wigner approach in the limit (44). We expand the classical field in powers of $1 / \sqrt{N}$ :

$$
\psi=\sqrt{N} \psi^{(0)}+\psi^{(1)}+\frac{1}{\sqrt{N}} \psi^{(2)}+\ldots
$$

where the $\psi^{(j)}$ are of the order of unity. We immediately note that the leading term of this expansion corresponds to a pure condensate with $N$ particles so that $\psi^{(0)}$ is simply the solution of the time-dependent Gross-Pitaevskii equation (47), $\psi^{(0)}=\phi$. This physically clear fact will be checked explicitly in what follows. In the initial thermal equilibrium state at time $t=0$ we expand (35) in powers of $1 / \sqrt{N}$ :

$$
\sqrt{N_{0}} \equiv \sqrt{N-\delta N}=\sqrt{N}-\frac{1}{2} \frac{\delta N}{\sqrt{N}}+\ldots
$$

so that we can identify explicitly:

$$
\begin{aligned}
& \psi^{(0)}(t=0)=\phi \\
& \psi^{(1)}(t=0)=\psi_{\perp} \\
& \psi^{(2)}(t=0)=-\frac{\delta N}{2} \phi+\phi_{\perp}^{(2)} .
\end{aligned}
$$


Following the same procedure as in the quantum case, we split each term of the expansion into a component along $\phi$ and a component orthogonal to $\phi$ :

$$
\psi^{(j)}(r)=\xi^{(j)} \phi(r)+\psi_{\perp}^{(j)}
$$

We calculate now the one-body density matrix $\rho$. Since we are using the Wigner representation for the atomic field on a finite spatial grid we have:

$$
\langle r|\hat{\rho}| s\rangle=\left\langle\psi^{*}(s) \psi(r)\right\rangle-\frac{1}{2 d V} \delta_{r, s}
$$

where $d V$ is the unit cell volume of the spatial grid and $\delta_{r, s}$ is a Kronecker $\delta$. Note that to simplify the notation we have omitted the subscript $W$ on the right hand side of the equation since the quantum and Wigner averages can be readily distinguished by the hats on the operators. We insert the expansions (52) and (57) into (58) and we use the fact that $\psi^{(0)}=\phi$ to obtain:

$$
\begin{aligned}
\langle r|\hat{\rho}| s\rangle_{T W} & =\phi^{*}(s) \phi(r)\left[N+\sqrt{N}\left\langle\xi^{(1)}+\xi^{(1) *}\right\rangle+\left\langle\left|\xi^{(1)}\right|^{2}\right\rangle+\left\langle\xi^{(2)}+\xi^{(2) *}\right\rangle-\frac{1}{2}\right] \\
& +\left\langle\psi_{\perp}^{(1) *}(s) \psi_{\perp}^{(1)}(r)\right\rangle-\frac{1}{2 d V} \mathcal{Q}_{r, s} \\
& +\phi^{*}(s)\left[\sqrt{N}\left\langle\psi_{\perp}^{(1)}(r)\right\rangle+\left\langle\xi^{(1) *} \psi_{\perp}^{(1)}(r)\right\rangle+\left\langle\psi_{\perp}^{(2)}(r)\right\rangle\right]+\{r \leftrightarrow s\}^{*} \\
& +O\left(\frac{1}{\sqrt{N}}\right)
\end{aligned}
$$

where we have collected the terms "parallel-parallel" in the first line, the terms "orthogonal-orthogonal" in the second line and the terms "orthogonal-parallel" in the third line, and where the matrix $\mathcal{Q}_{r, s} / d V=\delta_{r, s} / d V-\phi(r) \phi^{*}(s)$ is the discrete version of the projector $Q=1-|\phi\rangle\langle\phi|$. As we show in appendix E, by using the evolution equation of the field (11) and the initial conditions (54), (55) and(56) the following identities hold at all times:

$$
\begin{aligned}
\psi^{(0)} & =\phi \\
\sqrt{N}\left\langle\xi^{(1)}+\xi^{(1) *}\right\rangle+\left\langle\left|\xi^{(1)}\right|^{2}\right\rangle+\left\langle\xi^{(2)}+\xi^{(2) *}\right\rangle & =-\langle\delta \hat{N}\rangle \\
\left\langle\psi_{\perp}^{(1) *}(s) \psi_{\perp}^{(1)}(r)\right\rangle-\frac{1}{2 d V} \mathcal{Q}_{r, s} & =\left\langle\hat{\Lambda}^{\dagger}(s) \hat{\Lambda}(r)\right\rangle \\
\sqrt{N}\left\langle\psi_{\perp}^{(1)}(r)\right\rangle+\left\langle\xi^{(1) *} \psi_{\perp}^{(1)}(r)\right\rangle+\left\langle\psi_{\perp}^{(2)}(r)\right\rangle & =\phi_{\perp}^{(2)}(r) .
\end{aligned}
$$

As we have already mentioned the first identity (60) reflects the fact that at zero order in the expansion we have a pure condensate with $N$ particles evolving according to the time-dependent Gross-Pitaevskii equation. At time $t=0$ the three other identities are easily established since we have simply $\left\langle\psi_{\perp}^{(1)}\right\rangle=0, \xi^{(1)}=0$ and $\xi^{(2)}=-\delta N / 2$. At later times the mean value $\left\langle\psi_{\perp}^{(1)}\right\rangle$ remains equal to zero while $\xi^{(1)}$ develops a nonzero imaginary part corresponding to phase change of $\psi$ in the mode $\phi$ due to the interaction with the noncondensed particles

$$
\psi=\sqrt{N} \phi+\xi^{(1)} \phi+\ldots \simeq \sqrt{N} e^{\xi^{(1)} / \sqrt{N}} \phi+\ldots
$$

After averaging over all stochastic realisations, this random phase change contributes to the condensate depletion in (61) and to the correction $\phi^{(2)}$ to the condensate wavefunction in (63) [24]. As a consequence of the purely imaginary character of $\xi^{(1)}$ the quantity proportional to $\sqrt{N}$ in (61) vanishes. The identity (62) reflects the fact that in the linearised regime quantum fluctuations (here $\hat{\Lambda}$ ) and classical fluctuations (here $\psi_{\perp}^{(1)}$ ) around the Gross-Pitaevskii condensate field $\sqrt{N} \phi$, evolve according to the same equations. We find interestingly that the average $\left\langle\psi_{\perp}^{(2)}\right\rangle$ in (63) evolves under the influence of the mean field of the noncondensed particles, i.e. the Hartree-Fock term and the anomalous average contribution. In the Wigner representation the Hartree-Fock mean field term $2 g\left\langle\psi_{\perp}^{(1) *} \psi_{\perp}^{(1)}\right\rangle$ differs from the physical mean field $2 g\left\langle\hat{\Lambda}^{\dagger} \hat{\Lambda}\right\rangle$ by the term $g\left(1-|\phi|^{2} d V\right) / d V \simeq g / d V$. We note however that this brings in a global phase change of the condensate wavefunction having no effect on the one-body density matrix, and which is compensated anyway by the $-g / d V$ term in the Wigner drift term (16). In our calculations this is reflected by the fact that this term does not contribute to $\phi_{\perp}^{(2)}$. 
With the identities (60 63) we identify line by line the quantum expression (50) and the truncated Wigner expression (59) for the one-body density matrix of the system up to terms of $O(1)$ : these two expressions coincide apart from the term $1 / 2$ in the occupation number of the mode $\phi$. This slight difference $(1 / 2 \ll N)$ comes from the fact that in the initial sampling of the Wigner function in thermal equilibrium we have treated classically the condensate mode. These results establish the equivalence between the truncated Wigner method and the time-dependent Bogoliubov approach of [5] up to neglected terms $O(1 / \sqrt{N})$ in the limit (44).

Let us however come back to the expansions performed in the limit (44). We have mentioned that the small formal parameter is $1 / \sqrt{N}$ but we now wish to identify the small physical parameter of the expansion. In the quantum theory of [5] one gets the small parameter

$$
\epsilon_{\text {quant }}=\left(\frac{\langle\delta \hat{N}\rangle}{N}\right)^{1 / 2}
$$

where $\langle\delta \hat{N}\rangle$ is the Bogoliubov prediction for the number of noncondensed particles. In the expansion (52) of the evolving classical field we compare the norm of the first two terms, ignoring the field phase change $\xi^{(1)} \phi$ :

$$
\epsilon_{\mathrm{wig}}=\left(\frac{\left\langle d V \sum_{r}\left|\psi_{\perp}^{(1)}\right|^{2}\right\rangle}{N}\right)^{1 / 2}=\left(\frac{\langle\delta \hat{N}\rangle+(\mathcal{N}-1) / 2}{N}\right)^{1 / 2} .
$$

The validity condition of the expansion (52) in the truncated Wigner approach is then:

$$
N \gg\langle\delta \hat{N}\rangle, \mathcal{N} / 2
$$

which is more restrictive than in the quantum case. What indeed happens in the regime $\langle\delta \hat{N}\rangle \ll N<\mathcal{N} / 2$ ? We expect the truncated Wigner approach not to recover the predictions of the Bogoliubov approach of [5] which are correct in this limit. We therefore set a necessary condition for the validity of the truncated Wigner approach:

$$
N \gg \mathcal{N} / 2 \text {. }
$$

We interpret this condition as follows: the extra noise introduced in the Wigner representation (see discussion after (27)) contributes to the nonlinear term $g|\psi|^{2}$ in the evolution equation for the field; (68) means that this fluctuating additional mean field potential of order $g /(2 d V)$ should be much smaller than the condensate mean field of order $g N / V$ where $V=\mathcal{N} d V$ is the volume of the system. Condition (68) is also equivalent to $\rho d V \gg 1$, where $\rho$ is the atomic density, i.e. there should be on average more than one particle per grid site. We note that it is compatible with the conditions (8) on the spatial steps of the grid in the regime of a degenerate $\left(\rho \lambda^{3} \gg 1\right)$ and a weakly interacting $\left(\rho \xi^{3} \gg 1\right)$ Bose gas. Condition (68) is therefore generically not restrictive.

A last important point for this subsection is that the time-dependent Bogoliubov approach, relying on a linearisation of the field equations around a pure condensate solution, is usually restricted to short times in the case of an excited condensate, so it cannot be used to test the condition of validity of the truncated Wigner approach in the long time limit. It was found indeed in [25] that nonlinearity effects in the condensate motion can lead to a polynomial or even exponential increase in time of $\langle\delta \hat{N}\rangle$ which eventually invalidates the time-dependent Bogoliubov approach. The truncated Wigner approach in its full nonlinear version does not have this limitation however, as we have checked with a time-dependent $1 \mathrm{D}$ model in [3].

\section{B. Beliaev-Landau damping in the truncated Wigner approach}

In this section we consider a spatially homogeneous Bose condensed gas in a cubic box in three dimensions with periodic boundary conditions. We imagine that with a Bragg scattering technique we excite coherently a Bogoliubov mode of the stationary Bose gas, as was done experimentally at MIT [26, 27], and we study how the excitation decays in the Wigner approach due to Landau and Beliaev damping.

\section{Excitation procedure and numerical results}

We wish to excite coherently the Bogoliubov mode of wavevector $k_{0} \neq 0$. With a Bragg scattering technique using two laser beams with wave vector difference $q$ and frequency difference $\omega$ we induce a perturbation potential

$$
W=\int d^{3} r\left(\frac{W_{0}}{2} e^{i(q \cdot r-\omega t)}+c . c .\right)
$$


We match the wavevector and frequency of the perturbation to the wavevector $k_{0}$ and the eigenfrequency $\omega_{0}=\epsilon_{0} / \hbar$ of the Bogoliubov mode we wish to excite:

$$
q=k_{0} \quad \omega=\epsilon_{0} / \hbar=\omega_{0} .
$$

During the excitation phase, we expect that two Bogoliubov modes are excited from the condensate, the modes with wavevectors $k_{0}$ and $-k_{0}$. We anticipate the perturbative approach of next subsection which predicts that the mode of wavevector $k_{0}$, being excited resonantly, has an amplitude growing linearly with time, while the mode with wavevector $-k_{0}$, being excited off-resonance, has an oscillating amplitude vanishing periodically when $t$ is a multiple integer of $\pi / \omega_{0}$. In the truncated Wigner simulation we therefore stop the excitation phase at

$$
t_{\mathrm{exc}}=\frac{\pi}{\omega_{0}}
$$

We introduce the amplitudes of the classical field $\psi$ of the Bogoliubov modes. We first define the field

$$
\Lambda_{\text {static }}(r) \equiv \frac{1}{\sqrt{N}} a_{\phi}^{*} \psi_{\perp}(r)
$$

where $a_{\phi}$ and $\psi_{\perp}$ are the components of $\psi$ orthogonal and parallel to the static condensate wavefunction $\phi(r)=1 / L^{3 / 2}$ (see (18) ). The component along the Bogoliubov mode with wavevector $k$ is then

$$
b_{k}=d V \sum_{r} u_{k}^{*}(r) \Lambda_{\text {static }}(r)-v_{k}^{*}(r) \Lambda_{\text {static }}^{*}(r) .
$$

The functions $u_{k}$ and $v_{k}$ are plane waves with wavevector $k \neq 0$

$$
u_{k}(r)=\frac{1}{\sqrt{L^{3}}} U_{k} e^{i k \cdot r} \quad v_{k}(r)=\frac{1}{\sqrt{L^{3}}} V_{k} e^{i k \cdot r}
$$

and the real coefficients $U_{k}$ and $V_{k}$ are normalised to $U_{k}^{2}-V_{k}^{2}=1$ :

$$
U_{k}+V_{k}=\frac{1}{U_{k}-V_{k}}=\left(\frac{\hbar^{2} k^{2} / 2 m}{\hbar^{2} k^{2} / 2 m+2 \mu}\right)^{1 / 4}
$$

where the chemical potential is $\mu=g N / L^{3}$.

We denote by $b_{0}$ the amplitude of the field $\Lambda_{\text {static }}$ along the Bogoliubov mode of wavevector $k_{0}$, and $b_{-0}$ the amplitude along the mode with opposite wavevector. We show the mean values of these amplitudes as function of time obtained from the truncated Wigner simulation in figure 3 . In the initial thermal state these mean values vanish, and they become nonzero during the excitation phase due to the coherent excitation procedure. At later times they decay to zero again [28].

\section{Perturbative analysis of the truncated Wigner approach: Beliaev-Landau damping}

In the appendix $\mathrm{F}$ we report the exact equations of motion of the classical field $\Lambda_{\text {static }}$ defined by (72) in the truncated Wigner approach. We now make the assumption that $\Lambda_{\text {static }}$ is small compared with $\sqrt{N} \phi$, implying that

$$
N \gg\langle\delta \hat{N}\rangle, \frac{\mathcal{N}}{2}
$$

where $\langle\delta \hat{N}\rangle$ represents here the mean number of particles in the excited modes of the cubic box. In this regime we neglect terms which are at least cubic in $\Lambda_{\text {static }}$ in (F2) and we replace the number of particles in the ground state of the box by the total number of particles $N$, except in the zeroth order term in $\Lambda_{\text {static }}$ where we replace it by its initial mean value $\left\langle N_{0}\right\rangle$. We then find:

$$
\begin{aligned}
i \hbar \frac{d}{d t} \Lambda_{\text {static }} \simeq & \sqrt{\left\langle N_{0}\right\rangle} \mathcal{Q} h_{0} \phi+\mathcal{Q} h_{0} \Lambda_{\text {static }}+\frac{N g}{L^{3}}\left(\Lambda_{\text {static }}^{*}+2 \Lambda_{\text {static }}\right) \\
+ & \frac{g \sqrt{N}}{\sqrt{L^{3}}} \mathcal{Q}\left(\Lambda_{\text {static }} \Lambda_{\text {static }}+2 \Lambda_{\text {static }}^{*} \Lambda_{\text {static }}\right)-\frac{1}{\sqrt{N L^{3}}} \Lambda_{\text {static }}(r) d V \sum_{s} W_{0} \cos (q \cdot s-\omega t) \Lambda_{\text {static }}^{*}(s),
\end{aligned}
$$




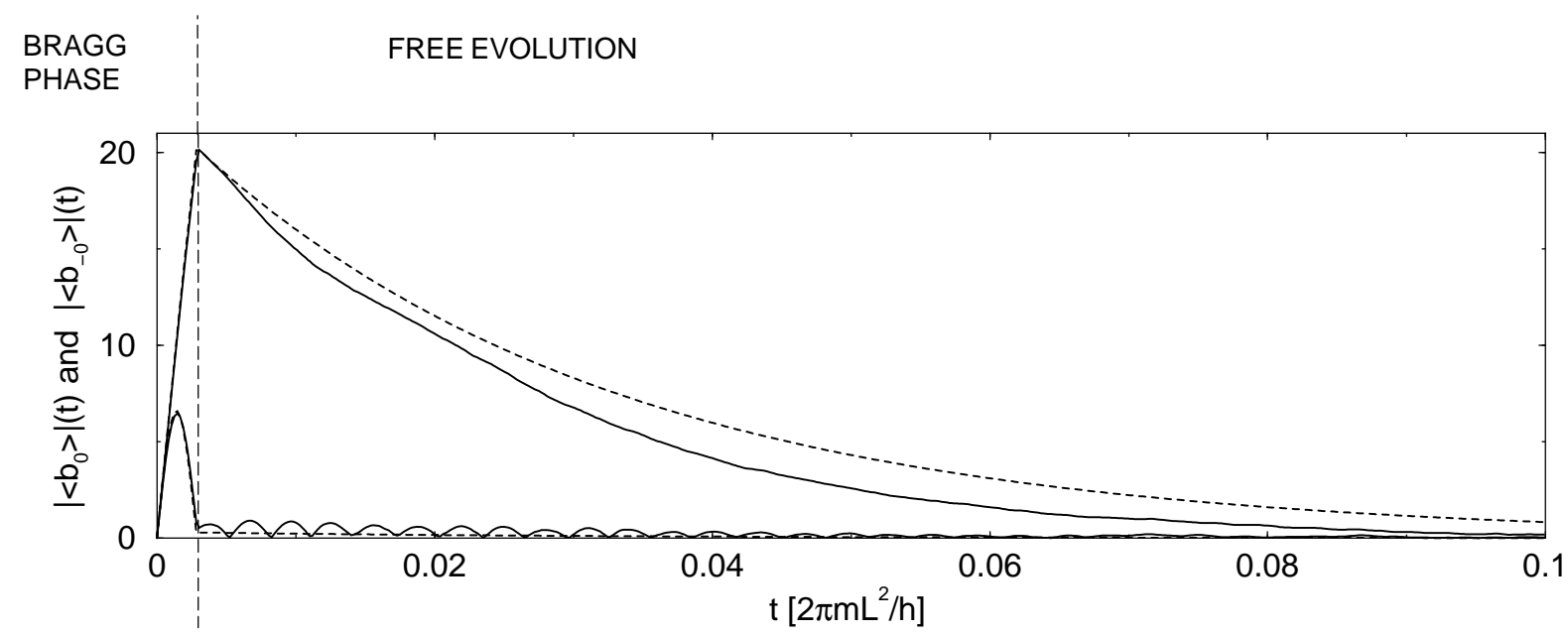

FIG. 3: Bragg excitation of a Bogoliubov mode of wavevector $k_{0}$ and frequency $\omega_{0}$ for a finite temperature Bose condensed gas in a cubic box. The vertical dashed line at time $t=\pi / \omega_{0}$ indicates the time after which the perturbation $W$ is discontinued. Solid lines: evolution of the field amplitudes of the Bogoliubov modes with wavevectors $k_{0}=(12 \pi / L, 0,0)$ (upper curve) and $-k_{0}$ (lower curve) in the Wigner simulation after averaging over 100 realizations. Only the mode $k_{0}$ is excited resonantly by Bragg scattering. After the coherent excitation Bragg phase, the amplitudes of the two modes are damped. Dashed line: perturbative approach of subsection IVB2 The truncated Wigner approach and the perturbation theory give comparable results. $N=5 \times 10^{4}, k_{B} T=3 \mu, \hbar \omega_{0}=2.2 \mu, W_{0}=0.175 \mu, \mu=500 \hbar^{2} / m L^{2}$. In the Wigner simulation a grid with 22 points per dimension is used, so that $\mathcal{N}=22^{3}=10648 \ll N$. In the perturbative approach a grid of 48 points per dimension is used to avoid truncation effects. The initial mean number of noncondensed particles is $N-\left\langle N_{0}\right\rangle \simeq 5000$.

where $W_{0}$ is non zero only during the excitation phase. In this equation $h_{0}=p^{2} / 2 m+W_{0} \cos (q \cdot r-\omega t)$ is the one-body part of the Hamiltonian including the kinetic energy and the Bragg excitation potential, and $\mathcal{Q}$ projects orthogonally to the static condensate mode $\phi$. The term of zeroth order in $\Lambda_{\text {static }}$ is a source term which causes $\Lambda_{\text {static }}$ to acquire a nonzero mean value during the evolution. The terms of first order in $\Lambda_{\text {static }}$ in (77) describe the evolution in the static Bogoliubov approximation. Terms of second order provide the damping we are looking for. We project equation (77) over the static Bogoliubov modes (74) by using:

$$
\Lambda_{\text {static }}(r)=\sum_{k \neq 0} b_{k} u_{k}(r)+b_{k}^{*} v_{k}^{*}(r)
$$

with the mode functions $u_{k}(r)$ and $v_{k}(r)$ defined in (74). Terms nonlinear in $\Lambda_{\text {static }}$ in (77) then correspond to an interaction between the Bogoliubov modes.

We assume that the excitation phase is much shorter than the damping time of the coherently excited mode. As a consequence we can neglect in this phase the processes involving interaction among the Bogoliubov modes. Also in the action of the perturbation $W$ we keep only the term acting on the condensate mode, that is the first term on the right hand side of (77), which is $\sqrt{\left\langle N_{0}\right\rangle}$ larger than the terms acting on the noncondensed modes. For the choice of parameters (70) only the two modes with wavevectors $k_{0}$ and $-k_{0}$ are excited from the condensate by the perturbation $W$; the amplitudes of the field in these modes evolve according to

$$
\begin{aligned}
i \hbar \frac{d}{d t} b_{0} & =\hbar \omega_{0} b_{0}+\sqrt{\left\langle N_{0}\right\rangle} \frac{W_{0}}{2}\left(U_{0}+V_{0}\right) e^{-i \omega_{0} t} \\
i \hbar \frac{d}{d t} b_{-0} & =\hbar \omega_{0} b_{-0}+\sqrt{\left\langle N_{0}\right\rangle} \frac{W_{0}}{2}\left(U_{0}+V_{0}\right) e^{i \omega_{0} t} .
\end{aligned}
$$

By integrating these equations we realise that the mean amplitude $\left\langle b_{0}\right\rangle$ grows linearly in time, since the mode is excited resonantly, while the mean amplitude $\left\langle b_{-0}\right\rangle$ oscillates and vanishes at $t=\pi / \omega_{0}$.

After the excitation phase we include the second order terms that provide damping:

$$
i \hbar \frac{d}{d t} b_{0}=\epsilon_{0} b_{0}+\sum_{i, j} A_{i, j}^{0} b_{i} b_{j}+\left(A_{i, 0}^{j}+A_{0, i}^{j}\right) b_{i}^{*} b_{j}+\sum_{i, j}\left(B_{i, j, 0}+B_{0, i, j}+B_{i, 0, j}\right) b_{i}^{*} b_{j}^{*}
$$

with

$$
A_{j, k}^{i}=\frac{g \sqrt{N}}{L^{3}}\left[U_{i}\left(U_{j}+V_{j}\right) U_{k}+\left(U_{i}+V_{i}\right) V_{j} U_{k}+V_{j}\left(U_{k}+V_{k}\right) V_{i}\right] \delta_{i, j+k}
$$




$$
B_{i, j, k}=\frac{g \sqrt{N}}{L^{3}} V_{i}\left(U_{j}+V_{j}\right) U_{k} \delta_{-i, j+k} .
$$

and where $i, j, k$ denote momenta. The last terms with the $B$ 's in (81) do not conserve the Bogoliubov energy and we can neglect them here for the calculation of the damping rate since we are going to use second order perturbation theory; we would have to keep them in order to calculate frequency shifts. In the terms with the $A$ 's we recognise two contributions: the term with $A_{i, j}^{0}$ describes a Beliaev process where the excited mode can decay into two different modes while the term with $A_{i, 0}^{j}+A_{0, i}^{j}$ describes a Landau process where the excited mode by interacting with another mode is scattered into a third mode [29]. We introduce the coefficients $\tilde{b}$ in the interaction picture

$$
\tilde{b}_{j}=b_{j} e^{i \epsilon_{j} t / \hbar}
$$

where $\epsilon_{j}$ is the Bogoliubov eigenenergy of the mode with wavevector $j$, and we solve (81) to second order of timedependent perturbation theory to obtain:

$$
\begin{aligned}
\left\langle\tilde{b_{0}}(t)-\tilde{b_{0}}(0)\right\rangle \simeq & -\frac{1}{\hbar^{2}} \sum_{i, j} A_{i, j}^{0}\left(A_{i, j}^{0}+A_{j, i}^{0}\right) I_{t}\left(\epsilon_{0}-\epsilon_{i}-\epsilon_{j}\right)\left(1+\bar{n}_{i}+\bar{n}_{j}\right)\left\langle\tilde{b_{0}}(0)\right\rangle \\
& -\frac{1}{\hbar^{2}} \sum_{i, j}\left(A_{i, 0}^{j}+A_{0, i}^{j}\right)^{2} I_{t}\left(\epsilon_{0}+\epsilon_{i}-\epsilon_{j}\right)\left(\bar{n}_{i}-\bar{n}_{j}\right)\left\langle\tilde{b}_{0}(0)\right\rangle \\
& -\frac{1}{\hbar^{2}} 2\left(A_{0,0}^{0+0}\right)^{2} I_{t}\left(\epsilon_{0}+\epsilon_{0}-\epsilon_{0+0}\right)\left\langle\tilde{b_{0}^{*}}(0) \tilde{b_{0}}(0) \tilde{b_{0}}(0)\right\rangle
\end{aligned}
$$

where $0+0$ represents the mode of wavevector $2 k_{0}$ and where

$$
\begin{aligned}
& I_{t}(\nu)=\int_{0}^{t} d \tau e^{i \nu \tau / \hbar} f_{\tau}(\nu) \\
& f_{\tau}(\nu)=\int_{0}^{\tau} d \theta e^{-i \nu \theta / \hbar} .
\end{aligned}
$$

The $\bar{n}_{j}$ 's are the occupation numbers of the Bogoliubov modes in thermal equilibrium given by the Bose formula

$$
\bar{n}_{j}=\frac{1}{e^{\epsilon_{j} / k_{B} T}-1}
$$

where $\epsilon_{j}$ is the energy of the Bogoliubov mode. In the language of nonlinear optics the last line in (85) describes a $\chi_{2}$ effect or a second harmonic generation which can be important if the conservation of energy condition $\epsilon_{2 k_{0}}=2 \epsilon_{k_{0}}$ is satisfied and if the initial amplitude $\left\langle\tilde{b}_{0}(0)\right\rangle=\beta$ is large since one has

$$
\left\langle\tilde{b_{0}^{*}}(0) \tilde{b_{0}}(0) \tilde{b_{0}}(0)\right\rangle=|\beta|^{2} \beta+\bar{n}_{0} 2 \beta .
$$

We have checked that the $\chi_{2}$ effect is negligible for the low amplitude coherent excitations considered in the numerical examples of this paper: $\epsilon_{0}$ is larger than $\mu$ so that $k_{0}$ is not in the linear part of the Bogoliubov spectrum and therefore the second harmonic generation process is not resonant. By using the fact that:

$$
\operatorname{Re} I_{t}(\nu)=\frac{1}{2}\left|f_{t}(\nu)\right|^{2}=\frac{2 \hbar^{2}}{\nu^{2}} \sin ^{2} \frac{\nu \tau}{2 \hbar} \equiv \pi \hbar t \delta_{t}(\nu)
$$

where $\delta_{t}(\nu)$ converges to a Dirac delta distribution in the large $t$ limit, we calculate the evolution of the modulus of the Bogoliubov mode amplitude

$$
\begin{aligned}
\frac{\left|\left\langle b_{0}(t)\right\rangle\right|-\left|\left\langle b_{0}(0)\right\rangle\right|}{\left|\left\langle b_{0}(0)\right\rangle\right|} \simeq & -\frac{\pi t}{\hbar} \sum_{i, j} A_{i, j}^{0}\left(A_{i, j}^{0}+A_{j, i}^{0}\right) \delta_{t}\left(\epsilon_{0}-\epsilon_{i}-\epsilon_{j}\right)\left(1+\bar{n}_{i}+\bar{n}_{j}\right) \\
& -\frac{\pi t}{\hbar} \sum_{i, j}\left(A_{i, 0}^{j}+A_{0, i}^{j}\right)^{2} \delta_{t}\left(\epsilon_{0}+\epsilon_{i}-\epsilon_{j}\right)\left(\bar{n}_{i}-\bar{n}_{j}\right) .
\end{aligned}
$$

This formula can be applied to a finite size box as it contains finite width $\delta$ 's. By plotting equation (91) as a function of time we can identify a time interval over which it is approximately linear in time, and we determine the slope $-\gamma_{\text {perturb }}$ with a linear fit [30]. Heuristically we then compare $\exp \left(-\gamma_{\text {perturb }} t\right)$ to the result of the truncated Wigner simulation, see figure 3 and we obtain a good agreement for this particular example [31].

In the thermodynamic limit, when the Bogoliubov spectrum becomes continuous, the discrete sums in (91) can be replaced by integrals and the finite width $\delta_{t}$ is replaced by a Dirac $\delta$ distribution. In this case an analytical expression for the damping rate can be worked out and we recover exactly the expression for the Beliaev and Landau damping rate obtained in the quantum field theory [32, 33,34$]$. 


\section{Validity condition of the truncated Wigner approach}

We now investigate numerically the influence of the grid size on the predictions of the truncated Wigner simulation. The line with squares in figure 4 shows the damping rate obtained from the Wigner simulation, defined as the inverse of the $1 / e$ half-width of $\left|\left\langle b_{0}(t)\right\rangle\right|$, as a function of the inverse grid size $1 / \mathcal{N}$. For small grids the results of the

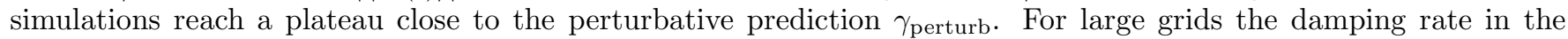

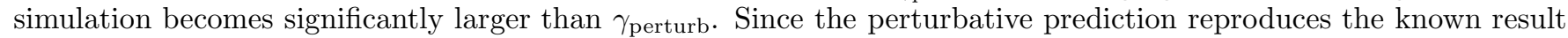
for Beliaev-Landau damping, we conclude that the results of the truncated Wigner simulation become incorrect for large grid sizes. The reason of such a spurious damping appearing in the Wigner simulation for large $\mathcal{N}$ will become clear below.

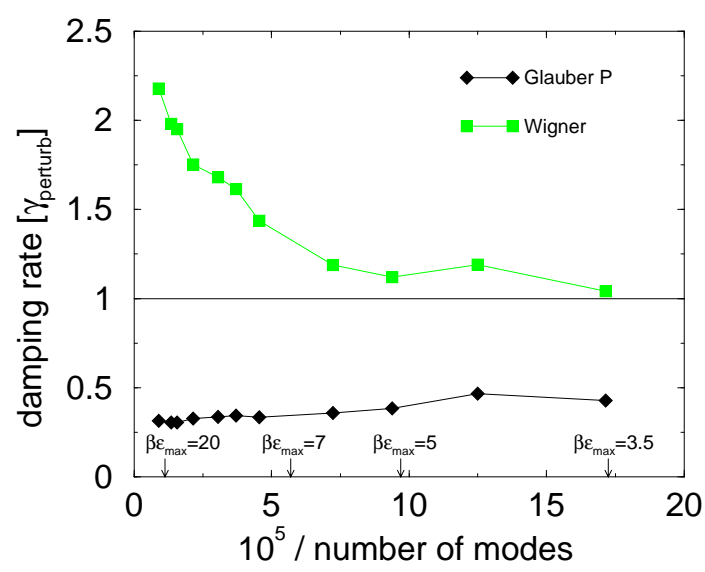

FIG. 4: Damping rate of the coherent excitation in the Bogoliubov mode of wavevector $k_{0}=(12 \pi / L, 0,0)$ and of frequency $\omega_{0}$ as a function of the inverse number of modes in the grid $1 / \mathcal{N}$ for the Glauber-P and the Wigner distributions. Each disk represents the average over 100 realisations of the simulation and the lines are a guide to the eye. $N=10^{5}, k_{B} T=3 \mu$, $\mu=500 \hbar^{2} / m L^{2}$, so that $\hbar \omega_{0}=2.2 \mu, \gamma_{\text {perturb }}^{-1}=0.061 m L^{2} / \hbar, W_{0}=0.0874 \mu$. The damping rate is expressed in units of

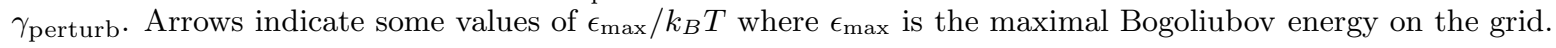

It is tempting to conclude from the perturbative calculation of subsection IVB2 that the validity condition of the truncated Wigner approach is dictated only by the condition $\mathcal{N} \ll N$. To check this statement we have performed a second set of simulations (not shown) for a particle number $N$ reduced by a factor of two keeping the size of the box $L$, the chemical potential $\mu=N g / L^{3}$ and the temperature fixed. If the condition of validity of the truncated Wigner approach involves only the ratio $N / \mathcal{N}$ the plateaux in the damping time should start at the same value of $N / \mathcal{N}$ for the two sets of simulations. However this is not the case, and we have checked that on the contrary, the two curves seem to depend on the number of modes only.

Another way to put it is that the condition to have agreement between the truncated Wigner simulation and the perturbation theory of section IVB2 is not (or not only) that the number of particles should be larger than the number of modes. There is in fact another "hidden" condition in the perturbative calculation which is the hypothesis that the occupation numbers of the Bogoliubov modes are constant during the evolution. In reality, even in absence of the Bragg perturbation, our initial state which reproduces the correct thermal distribution for the quantum Bose gas, is not stationary for the classical field evolution (1). The perturbative expression (91) holds indeed in the limit $N / \mathcal{N} \gg 1$, but the occupation numbers of the Bogoliubov modes, initially equal to the Bose formula $\bar{n}_{j}$, change in the course of the time evolution in the simulation and this affects the damping rate. This effect is neglected in the perturbative formula (91) and it is found numerically to take place on a time interval comparable to the damping time of the Bogoliubov coherent excitation as we show in figure 5 .

What it is expected to happen in the absence of external perturbation is that the classical field equation (11), in the three-dimensional cubic box geometry considered here, displays an ergodic behaviour leading to thermalisation of the classical field $\psi$ towards its equilibrium distribution [11, 12]. In the regime where the noncondensed fraction is small and the number of modes is smaller than $N$, we can approximately view the classical field as a sum of Bogoliubov oscillators $b_{k}$ weakly coupled by terms leading to the nonlinearities in (F2). In the equilibrium state for the classical field dynamics we then expect the occupation numbers of the Bogoliubov modes to be given by the equipartition formula:

$$
\left\langle b_{k}^{*} b_{k}\right\rangle_{\text {class }}=\frac{k_{B} T_{\text {class }}}{\epsilon_{k}}
$$




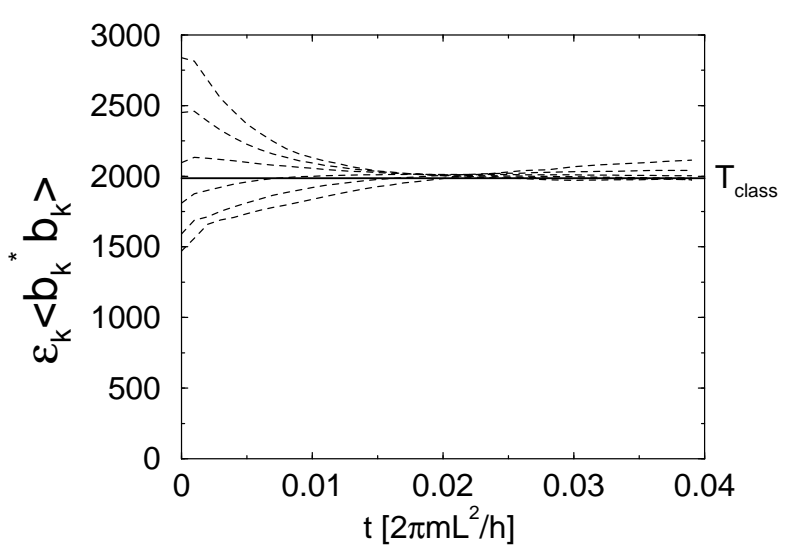

FIG. 5: Evolution of the squared amplitudes $\left\langle b_{k}^{*} b_{k}\right\rangle$ of the classical field Bogoliubov modes multiplied by the corresponding Bogoliubov energy $\epsilon_{k}$ in the truncated Wigner simulation in the absence of the Bragg perturbation. We have collected the Bogoliubov modes in energy channels of width $2 \mu$, so that the plotted quantity is the average among each channel of $\epsilon_{k}\left\langle b_{k}^{*} b_{k}\right\rangle$, with increasing energy from top to bottom at initial time $t=0$. The thick horizontal line is the expected temperature $T_{\text {class }}$ of the equilibrium classical field distribution as given by (94). Parameters are: $N=5 \cdot 10^{4}, k_{B} T=3 \mu, \mu=500 \hbar^{2} / m L^{2}$ and the vertical axis of the figure is in units of $\hbar^{2} / m L^{2}$, where $L$ is the cubic box size. The number of modes is 22 per spatial dimension so that the maximum Bogoliubov energy allowed on the grid is $\epsilon_{\max }=15.3 \mu$. The averaging in the simulation is performed over 500 realisations.

attributing a mean energy of $k_{B} T_{\text {class }}$ to each of the Bogoliubov mode. The classical field equilibrium temperature $T_{\text {class }}$ can then be deduced from the approximate conservation of the Bogoliubov energy [35]:

$$
\begin{aligned}
k_{B} T_{\text {class }} & =\frac{1}{\mathcal{N}-1} \sum_{k \neq 0} \epsilon_{k}\left\langle b_{k}^{*} b_{k}\right\rangle(t=0) \\
& =\frac{1}{\mathcal{N}-1} \sum_{k \neq 0}\left[\frac{\epsilon_{k}}{\exp \left(\beta \epsilon_{k}\right)-1}+\frac{1}{2} \epsilon_{k}\right] \\
& =\frac{1}{\mathcal{N}-1} \sum_{k \neq 0} \frac{\epsilon_{k}}{2 \tanh \left(\beta \epsilon_{k} / 2\right)} .
\end{aligned}
$$

The thermalisation of the Bogoliubov modes to the new temperature $T_{\text {class }}$ is nicely demonstrated in figure 5 , One sees that $\epsilon_{k}\left\langle b_{k}^{*} b_{k}\right\rangle$ indeed converges to a constant value almost independent of $k$. From the fact that $\tanh x<x$ for any $x>0$ we deduce that the classical equilibrium temperature $T_{\text {class }}$ is always larger than the real physical temperature $T$ of the gas. In the regime $k_{B} T \gg \mu$ this 'heating' increases the squared amplitudes $\left\langle b_{k}^{*} b_{k}\right\rangle$ of the modes of energy $\sim \mu$ by a factor $\simeq T_{\text {class }} / T$. Since the Landau damping rate is approximately proportional to the populations of these modes [32, 33, 34] the damping rate is increased roughly by a factor $T_{\text {class }} / T$, an artifact of the truncated Wigner approximation.

It is clear that $T_{\text {class }}$ will remain very close to $T$ as long as the maximum Bogoliubov energy allowed in the simulation remains smaller than $k_{B} T$. One can indeed in this case expand (94) in powers of $\beta \epsilon_{k}$. One has to expand the hyperbolic tangent up to cubic order to get a nonzero correction:

$$
\frac{T_{\text {class }}}{T} \simeq 1+\frac{1}{\mathcal{N}-1} \sum_{k \neq 0} \frac{\left(\beta \epsilon_{k}\right)^{2}}{12}
$$

The absence of terms of order $\beta \epsilon_{k}$ in (95) is a fortunate consequence of the noise added to the field in the Wigner representation. This added noise shifts the average $\left\langle b_{k}^{*} b_{k}\right\rangle(t=0)$ by $1 / 2$ with respect to the Bose formula.

When the maximum Bogoliubov energy becomes much larger than $k_{B} T$ we expect $T_{\text {class }}$ to become significantly larger than $T$. This is illustrated in figure 6 obtained by a numerical calculation of the sum in (94) for increasing grid sizes. We have also plotted in this figure the value that one would obtain for $T_{\text {class }}$ in the absence of the added Wigner noise (i.e. in a Glauber-P approach), that is by removing the terms $\epsilon_{k} / 2$ in (93). The Glauber-P distribution for the field $\psi$ in the sense of [36] is given by

$$
\psi=N_{0} \phi+\sum_{k \neq 0} b_{k} u_{k}+b_{k}^{*} v_{k}^{*}
$$


where the $b_{k}$ are chosen from a Gaussian distribution such that $\left\langle b_{k}^{*} b_{k}\right\rangle=1 /\left(\exp \left(\beta \epsilon_{k}\right)-1\right)$ and the value of $N_{0}$ is dictated by the normalisation condition $\|\psi\|^{2}=N$. In this case $T_{\text {class }}$ is always smaller than $T$, and deviates from $T$ for smaller grid sizes, since the fortunate cancellation of the order $\beta \epsilon_{k}$ obtained in (95) does not occur anymore. We expect in this case a spurious reduction of the damping rate. We have checked it by evolving an ensemble of fields of the form (96) with the Gross-Pitaevskii equation and we found that the damping rate is always smaller than half of the correct result even for the smallest grids that we tested, see the line with diamonds in figure 4 ,

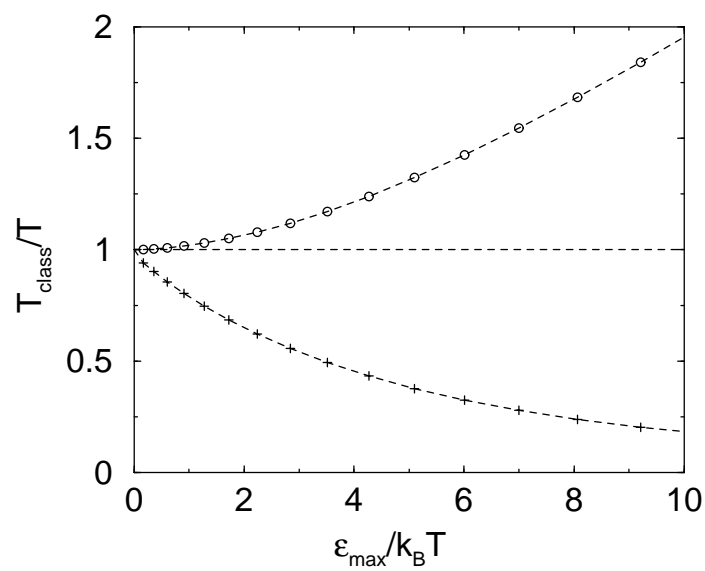

FIG. 6: Equilibrium temperature $T_{\text {class }}$ of the classical gas as function of the maximum energy $\epsilon_{\max }$ of the Bogoliubov modes on the momentum grid with the assumption of equipartition of the energy in the Bogoliubov modes. Circles: the initial field distribution is the Wigner distribution for the quantum gas at temperature T. Crosses: Glauber-P distribution defined in [36], amounting to the removal of the added Wigner noise from the initial field distribution. The dashed lines are a guide to the eye. The number of momentum components along each dimension of space goes from 2 to 30 in steps of 2 . The chemical potential is $\mu=500 \hbar^{2} / m L^{2}$ and the temperature is $k_{B} T=3 \mu$.

\section{CONCLUSION}

We have considered a possible way of implementing the truncated Wigner approximation to study the time evolution of trapped Bose-Einstein condensates perturbed from an initial finite temperature equilibrium state. First a set of random classical fields $\psi$ is generated to approximately sample the initial quantum thermal equilibrium state of the gas, in the Bogoliubov approximation assuming a weakly interacting and almost pure Bose-Einstein condensate. Then each field $\psi$ is evolved in the classical field approximation, that is according to the time-dependent Gross-Pitaevskii equation, with the crucial difference with respect to the more traditional use of the Gross-Pitaevskii equation that the field $\psi$ is now the whole matter field rather than the field in the mode of the condensate.

The central part of this paper is the investigation of the validity conditions of this formulation of the truncated Wigner approximation.

For short evolution times of the fields $\psi$ the dynamics of the noncondensed modes, i.e. the components of the field orthogonal to the condensate mode, is approximately linear; we can then use the time-dependent Bogoliubov approximation, both for the exact quantum problem and for the truncated Wigner approach. A necessary condition for the truncated Wigner approach to correctly reproduce the quantum results is then

$$
N \gg \mathcal{N} / 2
$$

where $\mathcal{N}$ is the number of modes in the Wigner approach and $N$ is the total number of particles in the gas. This condition can in general be satisfied in the degenerate and weakly interacting regime without introducing truncation effects due to a too small number of modes.

For longer evolution times the nonlinear dynamics of the noncondensed modes comes into play. When the classical field dynamics generated by the Gross-Pitaevskii equation is ergodic, e.g. in the example of a three dimensional gas in a cubic box considered in this paper, the set of Wigner fields $\psi$ evolves from the initial distribution mimicking the thermal state of the quantum gas at temperature $T$ to a classical field equilibrium distribution at temperature $T_{\text {class }}$. Since noise is added in the Wigner representation in all modes of the classical field to mimic quantum fluctuations it turns out that $T_{\text {class }}$ is always larger than $T$. If $T_{\text {class }}$ deviates too much from $T$ the truncated Wigner approximation can give incorrect predictions. For example we have found that the Beliaev-Landau damping of a Bogoliubov mode in 
the box, taking place with a time scale comparable to that of the 'thermalisation' of the classical field, is accelerated in a spurious way as the classical field 'warms up'. A validity condition for the truncated Wigner approach in this long time regime is therefore

$$
\left|T_{\text {class }}-T\right| \ll T \text {. }
$$

This condition sets a constraint on the maximum energy of the Bogoliubov modes $\epsilon_{\max }$ in the Wigner simulation: $\epsilon_{\max }$ should not exceed a few $k_{B} T$. More precisely one can use the following inequality to estimate the error [37]:

$$
\frac{\left|T_{\text {class }}-T\right|}{T}<\frac{1}{12} \frac{\left\langle\epsilon_{k}^{2}\right\rangle}{\left(k_{B} T\right)^{2}}<\frac{1}{12}\left(\frac{\epsilon_{\max }}{k_{B} T}\right)^{2}
$$

where $\left\langle\epsilon_{k}^{2}\right\rangle$ is the arithmetic mean of the squares of all the Bogoliubov energies in the Wigner simulation.

The fact that the initial set of Wigner fields is nonstationary under the classical field evolution could be a problem: the time-dependence of the observables could be affected in an unphysical way during the thermalisation to a classical distribution of the ensemble. To avoid this, we could start directly from the thermal equilibrium classical distribution [11, 13], restricting to the regime $\epsilon_{\max }<k_{B} T$.

A remarkable feature of the Wigner simulation is that $T_{\text {class }}$ deviates from $T$ at low values of $\epsilon_{\max }$ only quadratically in $\epsilon_{\max } / k_{B} T$. This very fortunate feature originates from the added noise in the Wigner representation. It explains why for $\epsilon_{\max }$ as high as $3.5 k_{B} T$ the truncated Wigner approach can still give very good results for the BeliaevLandau damping time (see Fig. 4). In contrast, if we remove the Wigner added noise, in the so-called Glauber-P representation, or if we add more noise, in the so-called Q representation, $T_{\text {class }}$ deviates from $T$ linearly in $\epsilon_{\max } / k_{B} T$. In this case we expect that the condition of validity of the classical Gross-Pitaevskii equation will be that all modes in the problem must be highly occupied, resulting in the stringent condition $\epsilon_{\max }<k_{B} T$. We therefore conclude that the Wigner representation is the most favorable representation of the quantum density operator with which to perform the classical field approximation. This fact, known in quantum optics for few mode systems, was not obvious for the highly multimode systems that are the finite temperature Bose gases.

Still, condition (98) is a serious limitation of the truncated Wigner method for simulating general ergodic three dimensional systems. One possibility to overcome this limitation is to proceed as in [38, 39] i.e. to treat the high energy modes as a reservoir, which leads to the inclusion of a stochastic term in the Gross-Pitaevskii equation. The advantage of this treatment is that the additional term has dissipative effects and thermalises the system to the correct quantum field thermal distribution in the stationary state as opposed to the classical one. However, one of the conceptual advantages of the truncated Wigner method and of classical field methods in general [9, 10, 11, 12] which we would like to keep is that apparent damping and irreversibility arise from the dynamics of a conservative equation (the Gross-Pitaevskii or nonlinear Schrödinger equation) as is the case in the original Hamiltonian equations for the quantum field.

Laboratoire Kastler Brossel is a research unit of École Normale Supérieure and of Université Pierre et Marie Curie, associated to CNRS. We acknowledge very useful discussions with Crispin Gardiner. This work was partially supported by National Computational Science Alliance under DMR $990016 \mathrm{~N}$ and used the NCSA SGI/CRAY Origin2000.

\section{APPENDIX A: BARE VS EFFECTIVE COUPLING CONSTANT}

In this appendix we describe how to adjust the potential $\mathcal{V}(r)$ defined on the grid in the simulation in order to reproduce correctly the low energy scattering properties of the true interatomic potential.

We start with the Schrödinger equation for a scattering state $\phi(r)$ of the discrete delta potential $\mathcal{V}(r) \equiv\left(g_{0} / d V\right) \delta_{r, 0}$ on the spatial grid of size $L_{\nu}$ and volume $V$ :

$$
\epsilon \phi(r)=\left(\frac{p^{2}}{m} \phi\right)(r)+\frac{g_{0}}{d V} \phi(r) \delta_{r, 0}
$$

where $m$ is twice the reduced mass and where $\phi(0)$ is different from zero. We project this equation on plane waves of momentum $k$ :

$$
\tilde{\phi}(k)=\frac{g_{0}}{V^{1 / 2}} \frac{\phi(0)}{\epsilon-\hbar^{2} k^{2} / m},
$$

where $\tilde{\phi}(k)$ is the component of $\phi$ on the plane wave $e^{i k \cdot r} / \sqrt{V}$. Fourier transforming back gives $\phi(0)$; dividing the resulting equation by $\phi(0)$ leads to the quantization condition

$$
1=\frac{1}{V} \sum_{k} \frac{g_{0}}{\epsilon-\hbar^{2} k^{2} / m}
$$


We define the effective coupling constant $g_{\text {eff }}$ in such a way that the energy of the lowest scattering state of the pseudopotential $g_{\text {eff }} \delta(r) \partial_{r}(r \cdot)$ in the box is the same as the energy of the lowest scattering state solution of (A3).

We now restrict ourselves to the case where the size of the box is much larger than the scattering length associated with $g_{\text {eff }}$. In this case the energy of the lowest scattering state for the continuous theory with the pseudopotential is very close to $g_{\text {eff }} / V$, so that we can calculate $g_{\text {eff }}$ from the equation $\epsilon=g_{\text {eff }} / V$. In this large box case, one can then check that the energy $\epsilon$ is negligible as compared to $\hbar^{2} k^{2} / m$ except if $k=0$. This gives

$$
g_{\text {eff }}=\frac{g_{0}}{1+\frac{1}{V} \sum_{k \neq 0} \frac{g_{0}}{\hbar^{2} k^{2} / m}}
$$

which allows us to adjust $g_{0}$ in order to have $g_{\mathrm{eff}}=g \equiv 4 \pi \hbar^{2} a / m$ where $a$ is the scattering length of the true interatomic potential.

The sum over $k$ in the denominator can be estimated by replacing the sum by an integral over $k$ and is found to be on the order of $k_{\max } a_{0}$ where $g_{0}=4 \pi \hbar^{2} a_{0} / m$ and $k_{\max }$ is the maximum momentum on the grid. $g_{0}$ is therefore very close to $g_{\text {eff }}$ when condition (9) is satisfied, so that we can set $g_{0} \simeq g_{\text {eff }}=g$. In the opposite limit of a grid step size tending to zero one gets $g_{\text {eff }} \rightarrow 0$, and we recover the known fact that a delta potential does not scatter in the continuous limit. We would have to increase $g_{0}$ continuously up to infinity as the grid step size tended to zero, if we wanted to get a finite $g_{\text {eff }}$ in this limit.

\section{APPENDIX B: AN IMPROVED BROWNIAN MOTION SIMULATION}

$A$ better choice for $\alpha$ and $Y$ - In our previous work [4] the drift matrix $\alpha$ and the noise matrix $Y$ were the hyperbolic sine and cosine of $\mathcal{L} /\left(2 k_{B} T\right)$, which imposed a time step $d t$ in the simulation which was exponentially small in the parameter $\epsilon_{\max } /\left(k_{B} T\right)$, where $\epsilon_{\max }$ is the largest eigenvalue of $\mathcal{L}$ allowed on the spatial grid of the simulation. We have now identified a choice that does not have this disadvantage:

$$
\begin{aligned}
\alpha & =2 M \\
Y & =\left(\begin{array}{cc}
\mathcal{Q} & 0 \\
0 & \mathcal{Q}^{*}
\end{array}\right),
\end{aligned}
$$

where the projector $\mathcal{Q}$ is defined in (25). With this new choice for $\alpha$ and $Y$ both the friction matrix and the noise matrix are bounded from above by unity, which allows a much larger $d t$ in the case $\epsilon_{\max }>k_{B} T$. To calculate the action of matrix $\alpha$ on the vector $\left(\psi_{\perp}, \psi_{\perp}^{*}\right)$ we write the hyperbolic tangent as:

$$
\tanh x=x \frac{\tanh x}{x} \equiv x F\left(x^{2}\right)
$$

The function $F(u)$ is then expanded on Chebyshev polynomials in the interval $u \in\left[0,\left(\epsilon_{\max } /\left(2 k_{B} T\right)\right)^{2}\right]$ and approximated by a polynomial of a given degree, typically 15 for $\epsilon_{\max } /\left(2 k_{B} T\right)=3$ and 25 for $\epsilon_{\max } /\left(2 k_{B} T\right)=6$, obtained by truncating a Chebyshev expansion of degree 50 [40].

An improved integration scheme - Initially we set $\psi_{\perp}=0$. Since the noise $d \xi$ is Gaussian, and because the stochastic differential equation (28) is linear, the probability distribution of $\psi_{\perp}$ is guaranteed to be Gaussian at any step of the integration so that the issue of the convergence of the distribution to the correct steady state distribution (21) can be discussed in terms of the convergence of the covariance matrix of the distribution to its right steady state value. Two issues in particular should be addressed: the error introduced by the discretisation in time (finite time step $d t$ of integration), and the error introduced by the integration over a finite time interval (approach to the steady state distribution).

We now explain how to face the first problem with an efficient integration scheme yielding an error on the steady state covariance matrix of the distribution scaling as $d t^{2}$, rather than $d t$ for the simple Euler scheme. In the numerical scheme the vector $\vec{X} \equiv\left(\psi_{\perp}, \psi_{\perp}^{*}\right)$ that stores the values of the field $\psi_{\perp}$ and of its complex conjugate $\psi_{\perp}^{*}$ on the discrete grid obeys the recursion relation:

$$
\vec{X}_{[t=(n+1) d t]}=\left(1-\alpha_{\text {num }} d t\right) \vec{X}_{[t=n d t]}+Y_{\text {num }}\left(\begin{array}{c}
d \xi_{[t=n d t]} \\
d \xi_{[t=n d t]}^{*}
\end{array}\right)
$$

with the initial condition $\vec{X}_{[t=0]}=0$. In this recursion relation the friction matrix $\alpha_{\text {num }}$ and the noise matrix $Y_{\text {num }}$ may differ from $\alpha$ and $Y$ of the continuous stochastic differential equation (28) by terms linear in $d t$ that remain to be determined in order to achieve an error scaling as $d t^{2}$. 
As we have already mentioned $\vec{X}_{[t=n d t]}$ is a Gaussian vector for any step $n$ of the iteration so that its probability distribution is characterised by the covariance matrix $C_{i j}^{(n)}=\left\langle X_{i} X_{j}^{*}\right\rangle$, with indices $i, j$ ranging from 1 to $2 \mathcal{N}$. From (B4) the covariance matrices are shown to obey the recursion relation:

$$
C^{(n+1)}=\left(1-\alpha_{\text {num }} d t\right) C^{(n)}\left(1-\alpha_{\text {num }}^{\dagger} d t\right)+\frac{2 d t}{d V} Y_{\text {num }} Y_{\text {num }}^{\dagger}
$$

For a small enough time step $d t$ this matrix sequence converges to a finite covariance matrix solving

$$
C^{(\infty)}=\left(1-\alpha_{\text {num }} d t\right) C^{(\infty)}\left(1-\alpha_{\text {num }}^{\dagger} d t\right)+\frac{2 d t}{d V} Y_{\text {num }} Y_{\text {num }}^{\dagger}
$$

We now try to choose the friction matrix and the noise matrix in order to minimise the deviation of $C^{(\infty)}$ from the desired value, which is the covariance matrix of the exact distribution (21), equal to $(2 M d V)^{-1}$. We look for $\alpha_{\text {num }}$ and $Y_{\text {num }}$ differing from the theoretical values (B1B2) by terms linear in $d t$, and leading to a covariance matrix different from the theoretical one by terms quadratic in $d t$ :

$$
\begin{aligned}
\alpha_{\text {num }} & =2 M+\alpha_{1} d t \\
Y_{\text {num }} & =\left(\begin{array}{cc}
\mathcal{Q} & 0 \\
0 & \mathcal{Q}^{*}
\end{array}\right)+Y_{1} d t \\
C^{(\infty)} & =\frac{1}{2 M d V}+O\left(d t^{2}\right) .
\end{aligned}
$$

Equation (B6) is satisfied up to order $d t$ irrespectively of the choice of $\alpha_{1}, Y_{1}$. Requiring that equation (B6) is satisfied up to order $d t^{2}$ leads to the condition

$$
-\alpha_{1} \frac{1}{4 M}-\frac{1}{4 M} \alpha_{1}+Y_{1}\left(\begin{array}{cc}
\mathcal{Q} & 0 \\
0 & \mathcal{Q}^{*}
\end{array}\right)+\left(\begin{array}{cc}
\mathcal{Q} & 0 \\
0 & \mathcal{Q}^{*}
\end{array}\right) Y_{1}^{\dagger}+M=0
$$

A particular solution of this equation is provided by $\alpha_{1}=0$ and $Y_{1}=Y_{1}^{\dagger}=-M / 2$. Our improved integration scheme is therefore

$$
\begin{aligned}
\alpha_{\text {num }} & =2 M \\
Y_{\text {num }} & =\left(\begin{array}{cc}
\mathcal{Q} & 0 \\
0 & \mathcal{Q}^{*}
\end{array}\right)-\frac{1}{2} M d t .
\end{aligned}
$$

The analysis of the recursion relation (B5) is easily performed for our improved integration scheme (B11 B12) since $\alpha_{\text {num }}, \alpha_{\text {num }}^{\dagger}, Y_{\text {num }}$ and hence $C^{(n)}$ are polynomials of $M$ and commute with $M$. As a consequence $C^{(\infty)}$ also commutes with $M$.

Let us first estimate the deviation of $C^{(\infty)}$ from the exact covariance matrix $(2 M d V)^{-1}$ :

$$
\begin{aligned}
C^{(\infty)} & =\left[1-\left(1-\alpha_{\mathrm{num}} d t\right)^{2}\right]^{-1} \frac{2 d t}{d V} Y_{\mathrm{num}} Y_{\mathrm{num}}^{\dagger} \\
& \simeq \frac{1}{2 M d V}\left[1+\frac{d t^{2}}{4} M^{2}+O\left(d t^{3}\right)\right]
\end{aligned}
$$

Because $M$ is bounded from above by unity we take in practice $d t=1 / 8$ so that the error is less than 0.5 percent.

Let us finally estimate the convergence time of the covariance matrices. The recursion relation (B5) can be rewritten as

$$
C^{(n+1)}-C^{(\infty)}=\left(1-\alpha_{\text {num }} d t\right)^{2}\left[C^{(n)}-C^{(\infty)}\right]
$$

so that the relative deviation of $C^{(n)}$ from its asymptotic value evolves as $\left(1-2 M_{\min } d t\right)^{2 n}$ where $M_{\min }$ is the smallest eigenvalue of $M$, that can be evaluated along the lines of [4]. We choose the number of time steps $n$ so that the relative deviation of $C^{(n)}$ from $C^{(\infty)}$ is less than 0.5 percent. 


\section{APPENDIX C: MOMENTS OF $N_{0}$ OF A HARMONICALLY TRAPPED IDEAL BOSE CONDENSED GAS}

We explain how to calculate the approximate expressions (42) for the moments of the number of condensed particles for an ideal Bose gas in an isotropic harmonic potential of frequency $\omega$ in the temperature regime $k_{B} T \gg \hbar \omega$ and in the Bogoliubov approximation. The calculation of the moments involves sums over the excited harmonic levels, see (41). By using the known degeneracy of the harmonic eigenstate manifold of energy $n \hbar \omega$ above the ground state energy the calculation reduces to the evaluation of sums of the form

$$
S_{p, q}(\epsilon)=\sum_{n=1}^{\infty} \frac{n^{p}}{(\exp (n \epsilon)-1)^{q}}
$$

where $\epsilon=\hbar \omega / k_{B} T$ is tending to zero, and the exponents $p$ and $q$ are positive integers.

First case: $q-p>1$ : In the limit $\epsilon \rightarrow 0$ the sum is dominated by the contribution of small values of $n$. Replacing $\exp (\epsilon n)-1$ by its first order expression we obtain:

$$
S_{p, q}(\epsilon) \simeq \frac{1}{\epsilon^{q}} \sum_{n=1}^{\infty} \frac{1}{n^{q-p}}=\frac{1}{\epsilon^{q}} \zeta(q-p)
$$

where $\zeta(\alpha)=\sum_{n>1} 1 / n^{\alpha}$ is the Riemann Zeta function.

Second case: $q-p<1$ : In the limit $\epsilon \rightarrow 0$ the contribution to the sum is dominated by large values of $n$. We then replace the discrete sum by an integral over $n$ from 1 to $+\infty$. Taking as integration variable $u=\epsilon n$ we arrive at

$$
S_{p, q}(\epsilon) \simeq \frac{1}{\epsilon^{p+1}} \int_{\epsilon}^{+\infty} d u \frac{u^{p}}{(\exp (u)-1)^{q}} .
$$

We can take the limit $\epsilon \rightarrow 0$ in the lower bound of the integral since $q-p<1$ :

$$
S_{p, q}(\epsilon) \simeq \frac{1}{\epsilon^{p+1}} I_{p, q}
$$

To calculate the resulting integral $I_{p, q}$ we expand the integrand in series of $\exp (-u)$ and integrate term by term over $u$ :

$$
I_{p, q} \equiv \int_{0}^{+\infty} d u \frac{u^{p}}{(\exp (u)-1)^{q}}=\sum_{k=0}^{\infty} \frac{p !}{(k+q)^{p+1}} \frac{(k+q-1) !}{k !(q-1) !}
$$

which can be expressed in terms of the Riemann Zeta function, e.g. $I_{2,2}=2(\zeta(2)-\zeta(3))$.

Third case: $q-p=1$ : In the limit $\epsilon \rightarrow 0$ both the small values of $n$ and the large values of $n$ contribute to the sum. We introduce a small parameter $\nu \ll 1$ that will be put to zero at the end of the calculation. For the summation indices $n<\nu / \epsilon$ we keep a discrete sum and we approximate each term of the sum by its first order expression in $\epsilon$, which is correct as $n \epsilon<\nu \ll 1$. For the summation indices $n>\nu / \epsilon$ we replace the sum by an integral, which is correct in the limit $\epsilon \rightarrow 0$ for a fixed $\nu$, since we then recognise a Riemann sum of a function with a converging integral. This leads to

$$
S_{p, p+1} \simeq \frac{1}{\epsilon^{p+1}}\left[\sum_{n=1}^{\nu / \epsilon} \frac{1}{n}+\int_{\nu}^{+\infty} d u \frac{u^{p}}{(\exp (u)-1)^{p+1}}\right] .
$$

In the limit $\epsilon \rightarrow 0$ the discrete sum is approximated by

$$
\sum_{n=1}^{\nu / \epsilon} \frac{1}{n} \simeq \log (\nu / \epsilon)+\gamma
$$

where $\gamma$ is Euler's constant. In the integral we remove and add $1 /(\exp (u)-1)$ to the integrand in order to get a convergent integrand which facilitates the calculation of the $\nu \rightarrow 0$ limit. The integral of $1 /(\exp (u)-1)$ can be calculated explicitly from the primitive $\log (1-\exp (-u))$ so that in the small $\nu$ limit

$$
\begin{aligned}
\int_{\nu}^{+\infty} d u \frac{u^{p}}{(\exp (u)-1)^{p+1}} & =\log \frac{1}{1-\exp (-\nu)}+\int_{\nu}^{+\infty} d u\left[\frac{u^{p}}{(\exp (u)-1)^{p+1}}-\frac{1}{\exp (u)-1}\right] \\
& \simeq-\log \nu+J_{p}
\end{aligned}
$$


where

$$
J_{p}=\int_{0}^{+\infty} d u\left[\frac{u^{p}}{(\exp (u)-1)^{p+1}}-\frac{1}{\exp (u)-1}\right] .
$$

The $-\log \nu$ term coming from the integral compensates the $\log \nu$ term coming from the sum in (C7) so that in the limit $\nu \rightarrow 0$ we get the $\nu$-independent estimate

$$
S_{p, p+1} \simeq \frac{1}{\epsilon^{p+1}}\left[-\log \epsilon+\gamma+J_{p}\right] .
$$

The quantity $J_{p}$ for $p>0$ can be calculated from a recursion relation obtained in the following way: we use the identity

$$
\frac{u^{p}}{(\exp (u)-1)^{p+1}}=-\frac{u^{p}}{(\exp (u)-1)^{p}}+u^{p} \frac{\exp (u)}{(\exp (u)-1)^{p+1}} .
$$

The first term of the above expression leads to an integral already calculated in (C5) and called $I_{p, p}$. We then integrate the second term of the above expression by parts, taking the derivate of $u^{p}$ with respect to $u$. This finally leads to

$$
J_{p}=J_{p-1}+\frac{1}{p}-I_{p, p} .
$$

We get in particular $J_{1}=1-\zeta(2)$ and $J_{2}=3 / 2-3 \zeta(2)+2 \zeta(3)$.

Finally we collect the approximations for the $S_{p, q}$ relevant for the calculation of the skewness of the number of condensed particles $N_{0}$ in $1 \mathrm{D}, 2 \mathrm{D}, 3 \mathrm{D}$ :

$$
\begin{array}{lll}
S_{0,1} \simeq \frac{-\log \epsilon+\gamma}{\epsilon} & S_{0,2} \simeq \frac{\zeta(2)}{\epsilon^{2}} & S_{0,3} \simeq \frac{\zeta(3)}{\epsilon^{3}} \\
S_{1,1} \simeq \frac{\zeta(2)}{\epsilon^{2}} & S_{1,2} \simeq \frac{-\log (\epsilon)+\gamma+1-\zeta(2)}{\epsilon^{2}} & S_{1,3} \simeq \frac{\zeta(2)}{\epsilon^{3}} \\
S_{2,1} \simeq \frac{2 \zeta(3)}{\epsilon^{3}} & S_{2,2} \simeq \frac{2 \zeta(2)-2 \zeta(3)}{\epsilon^{3}} & S_{2,3} \simeq \frac{-\log \epsilon+\gamma+J_{2}}{\epsilon^{3}}
\end{array}
$$

\section{APPENDIX D: EQUATIONS OF THE NUMBER CONSERVING BOGOLIUBOV APPROACH}

In this appendix we give the equations of motion for the operator $\hat{\Lambda}$ and for $\phi_{\perp}^{(2)}(r)$ from [5]. The evolution equation for $\hat{\Lambda}$ is:

$$
i \hbar \partial_{t}\left(\begin{array}{c}
\hat{\Lambda}(r, t) \\
\hat{\Lambda}^{\dagger}(r, t)
\end{array}\right)=\mathcal{L}(t)\left(\begin{array}{c}
\hat{\Lambda}(r, t) \\
\hat{\Lambda}^{\dagger}(r, t)
\end{array}\right)
$$

with $\mathcal{L}$ given by (24). The evolution equation for $\phi_{\perp}^{(2)}(r)$ is:

$$
\left(i \hbar \frac{d}{d t}-\mathcal{L}(t)\right)\left(\begin{array}{c}
\phi_{\perp}^{(2)}(t) \\
\phi_{\perp}^{(2) *}(t)
\end{array}\right)=\left(\begin{array}{r}
\mathcal{Q}(t) S(t) \\
-\mathcal{Q}^{*}(t) S^{*}(t)
\end{array}\right)
$$

where

$$
\begin{aligned}
S(r) & =-g N|\phi(r)|^{2} \phi(r)\left\langle 1+\sum_{s} d V \hat{\Lambda}^{\dagger}(s) \hat{\Lambda}(s)\right\rangle \\
& +2 g N \phi(r)\left\langle\hat{\Lambda}^{\dagger}(r) \hat{\Lambda}(r)\right\rangle+g N \phi^{*}(r)\langle\hat{\Lambda}(r) \hat{\Lambda}(r)\rangle \\
& -g N \sum_{s} d V|\phi(s)|^{2}\left\langle\left[\hat{\Lambda}^{\dagger}(s) \phi(s)+\hat{\Lambda}(s) \phi^{*}(s)\right] \hat{\Lambda}(r)\right\rangle .
\end{aligned}
$$




\section{APPENDIX E: TRUNCATED WIGNER APPROACH IN THE BOGOLIUBOV REGIME}

In this appendix we demonstrate the equivalences (60, 63). For convenience we change in this appendix the phase reference of the field $\psi$ which now evolves according to

$$
i \hbar \partial_{t} \psi=\left[p^{2} / 2 m+U(r, t)+g|\psi|^{2}-\mu\right] \psi
$$

where $\mu$ is the chemical potential in the time-independent Gross-Pitaevskii equation for the condensate wavefunction (17).

\section{Identification of the pure condensate wavefunction}

At $t=0$ equation (60) is satisfied. By keeping only terms of order $\sqrt{N}$ in (E1), in the limit (44), we obtain

$$
i \hbar \partial_{t} \psi^{(0)}=\left(h_{0}+g\left|\psi^{(0)}\right|^{2}-\mu\right) \psi^{(0)}
$$

where $h_{0}$ is the one-body part of the Hamiltonian. This shows that (60) holds at all times.

\section{2. "Orthogonal-orthogonal" contribution}

We wish to prove (62). To this aim we expand $\hat{\Lambda}$ and $\psi_{\perp}^{(1)}$ over the Bogoliubov modes:

$$
\begin{aligned}
\hat{\Lambda} & =\sum_{k} \hat{b}_{k} u_{k}+\hat{b}_{k}^{\dagger} v_{k}^{*} \\
\psi_{\perp}^{(1)} & =\sum_{k} b_{k} u_{k}+b_{k}^{*} v_{k}^{*}
\end{aligned}
$$

At $t=0$ the same mode functions $u_{k}$ and $v_{k}^{*}$ appear in the expansions of $\hat{\Lambda}$ and $\psi_{\perp}^{(1)}$. We wish to show that (E3 E4) hold at any time, or equivalently that $\hat{\Lambda}$ and $\psi_{\perp}^{(1)}$ have the same equations of motion. If we keep only terms of order $O(1)$ in (E1) we get

$$
i \hbar \partial_{t}\left(\begin{array}{c}
\psi^{(1)} \\
\psi^{(1) *}
\end{array}\right)=\mathcal{L}_{G P}\left(\begin{array}{c}
\psi^{(1)} \\
\psi^{(1) *}
\end{array}\right)
$$

where $\mathcal{L}_{G P}$ is the usual Bogoliubov operator obtained from (24) by eliminating all the projectors. By using the fact that

$$
\left(\begin{array}{c}
\psi_{\perp}^{(1)} \\
\psi_{\perp}^{(1) *}
\end{array}\right)=\left(\begin{array}{cc}
\mathcal{Q} & 0 \\
0 & \mathcal{Q}^{*}
\end{array}\right)\left(\begin{array}{c}
\psi^{(1)} \\
\psi^{(1) *}
\end{array}\right)
$$

and

$$
\left(\begin{array}{c}
\xi^{(1)} \phi \\
\xi^{(1) *} \phi^{*}
\end{array}\right)=\left(\begin{array}{cc}
\mathcal{P} & 0 \\
0 & \mathcal{P}^{*}
\end{array}\right)\left(\begin{array}{c}
\psi^{(1)} \\
\psi^{(1) *}
\end{array}\right)
$$

with the matrices

$$
\mathcal{P}_{r, s}=d V \phi(r) \phi^{*}(s) \quad \mathcal{Q}_{r, s}=\delta_{r, s}-d V \phi(r) \phi^{*}(s)
$$

we get

$$
\begin{aligned}
i \hbar \partial_{t}\left(\begin{array}{c}
\psi_{\perp}^{(1)} \\
\psi_{\perp}^{(1) *}
\end{array}\right) & =\mathcal{L}\left(\begin{array}{c}
\psi_{\perp}^{(1)} \\
\psi_{\perp}^{(1) *}
\end{array}\right)+\left(\xi^{(1)}+\xi^{(1) *}\right)\left(\begin{array}{cc}
\mathcal{Q} & 0 \\
0 & \mathcal{Q}^{*}
\end{array}\right)\left(\begin{array}{c}
g N|\phi|^{2} \phi \\
-g N|\phi|^{2} \phi^{*}
\end{array}\right) \\
i \hbar \frac{d}{d t} \xi^{(1)} & =d V \sum_{r} g N|\phi(r)|^{2}\left[\phi^{*}(r) \psi^{(1)}(r)+\psi^{(1) *}(r) \phi(r)\right] .
\end{aligned}
$$

The fact that the derivative of $\xi^{(1)}$ is purely imaginary and the initial condition $\xi^{(1)}=0$ guarantee that $\left(\xi^{(1)}+\xi^{(1) *}\right)=0$ for all times, which proves that $\hat{\Lambda}$ and $\psi_{\perp}^{(1)}$ have the same equations of motion. At all times we then have

$$
\left\langle\hat{\Lambda}^{\dagger}(s) \hat{\Lambda}(r)\right\rangle=\sum_{k} u_{k}(r) u_{k}^{*}(s)\left\langle\hat{b}_{k}^{\dagger} \hat{b}_{k}\right\rangle+v_{k}^{*}(r) v_{k}(s)\left\langle\hat{b}_{k} \hat{b}_{k}^{\dagger}\right\rangle
$$


and

$$
\left\langle\psi_{\perp}^{(1) *}(s) \psi_{\perp}^{(1)}(r)\right\rangle=\left\langle\hat{\Lambda}^{\dagger}(s) \hat{\Lambda}(r)\right\rangle+\frac{1}{2} \sum_{k} u_{k}(r) u_{k}^{*}(s)-v_{k}^{*}(r) v_{k}(s)
$$

where the amplitudes $b_{k}$ are time-independent and the $u_{k}, v_{k}$ are time-dependent modes evolving according to

$$
i \hbar \partial_{t}\left(\begin{array}{l}
u_{k} \\
v_{k}
\end{array}\right)=\mathcal{L}\left(\begin{array}{l}
u_{k} \\
v_{k}
\end{array}\right)
$$

By using the decomposition of unity, equation (61) of reference [5]:

$$
\sum_{k} u_{k}(r) u_{k}^{*}(s)-v_{k}^{*}(r) v_{k}(s)=\frac{1}{d V} \mathcal{Q}_{r, s}
$$

we prove (62).

\section{3. "Parallel-parallel" contribution}

We wish to prove (61). We use the fact that $\left\langle d V \sum_{r}|\psi(r)|^{2}\right\rangle$ is a constant of motion order by order in $1 / \sqrt{N}$. To order $\sqrt{N}$ we get

$$
\frac{d}{d t} N=0
$$

To order $N^{0}$ we get

$$
\frac{d}{d t}\left\langle\xi^{(1)}+\xi^{(1) *}\right\rangle=0
$$

which we verified directly in (E10). To order $1 / \sqrt{N}$ we get

$$
\frac{d}{d t}\left[\left\langle\xi^{(2)}+\xi^{(2) *}\right\rangle+\langle|\left(\left.\xi^{(1)}\right|^{2}\right\rangle+\left\langle d V \sum_{r}\left|\psi_{\perp}^{(1)}(r)\right|^{2}\right\rangle\right]=0 .
$$

Using (62) we then obtain

$$
\left\langle\xi^{(2)}+\xi^{(2) *}\right\rangle+\langle|\left(\left.\xi^{(1)}\right|^{2}\right\rangle+\langle\delta \hat{N}\rangle+\frac{\mathcal{N}-1}{2}=\text { constant } .
$$

At $t=0$ from (55), (56) we deduce

$$
\text { constant }=\frac{\mathcal{N}-1}{2}
$$

so that at any time

$$
\left\langle\xi^{(2)}+\xi^{(2) *}\right\rangle+\langle|\left(\left.\xi^{(1)}\right|^{2}\right\rangle=-\langle\delta \hat{N}\rangle
$$

Note that without the approximation in [3] we would have at $t=0$ constant $=\frac{\mathcal{N}}{2}$ and as a consequence $\left\langle\xi^{(2)}+\xi^{(2) *}\right\rangle+\langle|\left(\left.\xi^{(1)}\right|^{2}\right\rangle=-\langle\delta \hat{N}\rangle+\frac{1}{2}$. The contribution of the $1 / 2$ compensates exactly the term $-\frac{1}{2} \phi^{*}(s) \phi(r)$ in (59). We neglect here this contribution.

\section{Term "parallel-orthogonal"}

The last step consists in proving (63). We first remark that at $t=0\left\langle\psi_{\perp}^{(1)}\right\rangle=0$, and for linearity reasons $\left\langle\psi_{\perp}^{(1)}\right\rangle=0$ at all times. At $t=0$ (63) is satisfied by construction. We then have to deduce the equation of motion for

$$
\langle\chi\rangle \equiv\left\langle\xi^{(1) *} \psi_{\perp}^{(1)}+\psi_{\perp}^{(2)}\right\rangle
$$


and show that it coincides with the equation of motion for $\phi_{\perp}^{(2)}$. By keeping only terms of order $1 / \sqrt{N}$ in $(\underline{\text { E1) }}$ we get

$$
i \hbar \partial_{t}\left(\begin{array}{c}
\psi^{(2)} \\
\psi^{(2) *}
\end{array}\right)=\mathcal{L}_{G P}\left(\begin{array}{c}
\psi^{(2)} \\
\psi^{(2) *}
\end{array}\right)+\left(\begin{array}{r}
g N\left[\phi^{*} \psi^{(1) 2}+2 \phi\left|\psi^{(1)}\right|^{2}\right] \\
-g N\left[\phi \psi^{(1) * 2}+2 \phi^{*}\left|\psi^{(1)}\right|^{2}\right]
\end{array}\right)
$$

With a calculation analogous to the one we performed to obtain the derivative of $\left(\psi_{\perp}^{(1)}, \psi_{\perp}^{(1) *}\right)$, using (E18) to eliminate $\xi^{(2)}$ and replacing $\psi^{(1)}$ by $\xi^{(1)} \phi+\psi_{\perp}^{(1)}$, we obtain:

$$
\begin{aligned}
i \hbar \partial_{t}\left(\begin{array}{c}
\psi_{\perp}^{(2)} \\
\psi_{\perp}^{(2) *}
\end{array}\right) & =\mathcal{L}\left(\begin{array}{c}
\psi_{\perp}^{(2)} \\
\psi_{\perp}^{(2) *}
\end{array}\right)-\langle\delta \hat{N}\rangle\left(\begin{array}{c}
g N \mathcal{Q}|\phi|^{2} \phi \\
-g N \mathcal{Q}^{*}|\phi|^{2} \phi^{*}
\end{array}\right) \\
& +\left(\begin{array}{c}
g N \mathcal{Q}\left[2\left|\psi_{\perp}^{(1)}\right|^{2} \phi+2 \xi^{(1)} \phi^{2} \psi_{\perp}^{(1) *}+\phi^{*}\left(\psi_{\perp}^{(1)}\right)^{2}\right] \\
-g N \mathcal{Q}^{*}\left[2\left|\psi_{\perp}^{(1)}\right|^{2} \phi+2 \xi^{(1)} \phi^{2} \psi_{\perp}^{(1) *}+\phi^{*}\left(\psi_{\perp}^{(1)}\right)^{2}\right]^{*}
\end{array}\right) .
\end{aligned}
$$

In particular, we find that the terms involving $\left|\xi^{(1)}\right|^{2}$ disappear because $\left(\xi^{(1)}\right)^{2}=-\left|\xi^{(1)}\right|^{2}$. By using (E9) and (E10) we can calculate the derivative of $\langle\chi\rangle$ :

$$
i \hbar \partial_{t}\left(\begin{array}{c}
\langle\chi\rangle \\
\left\langle\chi^{*}\right\rangle
\end{array}\right)=\mathcal{L}\left(\begin{array}{c}
\langle\chi\rangle \\
\left\langle\chi^{*}\right\rangle
\end{array}\right)+\left(\begin{array}{c}
\mathcal{Q} R \\
-\mathcal{Q}^{*} R^{*}
\end{array}\right)
$$

with

$$
\begin{aligned}
R(r) & =-\langle\delta \hat{N}\rangle g N|\phi(r)|^{2} \phi(r)+2 g N \phi(r)\left[\left\langle\hat{\Lambda}^{\dagger} \hat{\Lambda}\right\rangle-\frac{1}{2}|\phi(r)|^{2}\right] \\
& +g N \phi^{*}\left\langle\hat{\Lambda}^{2}\right\rangle-g N\left\{\frac{1}{2} \phi(r)|\phi(r)|^{2}+d V \sum_{s}|\phi(s)|^{2}\left\langle\left[\hat{\Lambda}^{\dagger}(s) \phi(s)+\phi^{*}(s) \hat{\Lambda}(s)\right] \hat{\Lambda}(r)\right\rangle\right\}
\end{aligned}
$$

which is identical to (D3), except for the contribution of the term $1 / 2$ neglected in [3] as discussed after (E20). In order to obtain (E26) we used the identity (62) and the fact that all terms proportional to $\phi(r)$ are killed by the projector $\mathcal{Q}$ in (E25). Summarising, (E25) and (E26) together with $\left\langle\psi_{\perp}^{(1)}\right\rangle=0$ prove (63).

\section{APPENDIX F: EQUATION FOR THE NONCONDENSED FIELD IN THE WIGNER APPROACH}

In the truncated Wigner approach, we define the field $\Lambda_{\mathrm{ex}}(r)=a_{\phi}^{*} \psi_{\perp}(r) / \sqrt{N}$ where $\phi$ is at this stage an arbitrary wave function normalised to unity, $\psi_{\perp}$ is the component of $\psi$ orthogonal to $\phi$, and $a_{\phi}$ is the coefficient of $\psi$ along $\phi$. When $\psi$ solves the time-dependent Gross-Pitaevskii equation, the equation of motion for $\Lambda_{\text {ex }}$ is given by:

$$
i \hbar \frac{d \Lambda_{\mathrm{ex}}}{d t}=\frac{1}{\sqrt{N}} i \hbar \frac{d}{d t}\left(a_{\phi}^{*} \psi_{\perp}(r)\right)=d V \sum_{s} \sum_{k=0}^{4} \frac{R_{k}(r, s)}{N^{(k-1) / 2}}
$$

where we have collected the terms of the same power in $\Lambda_{\text {ex }}$ :

$$
\begin{aligned}
R_{0}(r, s) & =\frac{N_{\phi}}{N} \frac{\mathcal{Q}_{r, s}}{d V}\left[-i \hbar \partial_{t}+h_{0}+g N_{\phi}|\phi(s)|^{2}\right] \phi(s) \\
R_{1}(r, s) & =\frac{\mathcal{Q}_{r, s}}{d V}\left[h_{0}+2 g N_{\phi}|\phi(s)|^{2}\right] \Lambda_{\mathrm{ex}}(s)-\phi(r)\left(i \hbar \partial_{t} \phi^{*}(s)\right) \Lambda_{\mathrm{ex}}(s) \\
& +\frac{\mathcal{Q}_{r, s}}{d V} g N_{\phi} \phi^{2}(s) \Lambda_{\mathrm{ex}}^{*}(s)-\Lambda_{\mathrm{ex}}(r) \phi^{*}(s)\left(-i \hbar \partial_{t}+h_{0}+g N_{\phi}|\phi(s)|^{2}\right) \phi(s) \\
R_{2}(r, s) & =-\frac{N}{N_{\phi}} \Lambda_{\mathrm{ex}}^{*}(s) \Lambda_{\mathrm{ex}}(r)\left(-i \hbar \partial_{t}+h_{0}+2 g N_{\phi}|\phi(s)|^{2}\right) \phi(s) \\
& +g N \frac{\mathcal{Q}_{r, s}}{d V}\left[\Lambda_{\mathrm{ex}}^{2}(s) \phi^{*}(s)+2 \Lambda_{\mathrm{ex}}^{*}(s) \Lambda_{\mathrm{ex}}(s) \phi(s)\right] \\
& -g N \phi^{*}(s)|\phi(s)|^{2} \Lambda_{\mathrm{ex}}(s) \Lambda_{\mathrm{ex}}(r)
\end{aligned}
$$




$$
\begin{aligned}
R_{3}(r, s) & =g N \frac{N}{N_{\phi}}\left[\frac{\mathcal{Q}_{r, s}}{d V} \Lambda_{\mathrm{ex}}^{*}(s) \Lambda_{\mathrm{ex}}^{2}(s)-\Lambda_{\mathrm{ex}}^{* 2}(s) \Lambda_{\mathrm{ex}}(r) \phi^{2}(s)\right] \\
& -2 g N \frac{N}{N_{\phi}}|\phi(s)|^{2} \Lambda_{\mathrm{ex}}^{*}(s) \Lambda_{\mathrm{ex}}(s) \Lambda_{\mathrm{ex}}(r) \\
R_{4}(r, s) & =-g N\left(\frac{N}{N_{\phi}}\right)^{2} \Lambda_{\mathrm{ex}}^{* 2}(s) \Lambda_{\mathrm{ex}}(s) \Lambda_{\mathrm{ex}}(r) \phi(s)
\end{aligned}
$$

where $N_{\phi}=a_{\phi}^{*} a_{\phi}, h_{0}=p^{2} / 2 m+U(r, t)$ is the one-body part of the Hamiltonian and $\mathcal{Q}_{r, s}=\delta_{r, s}-d V \phi(r) \phi^{*}(s)$ projects orthogonally to $\phi$. In the case of a uniform wavefunction $\phi(r)=1 / L^{3 / 2}$ we have the following simplifications: (i) $\partial_{t} \phi$ is equal to zero, (ii) the constant terms like $|\phi(s)|^{2} \phi(s)$ are killed by the projectors, (iii) for terms having a vanishing spatial sum, $\frac{\mathcal{Q}_{r, s}}{d V}$ can be replaced by $\delta_{r, s}$, (iv) the sum over $s$ of $\psi_{\perp}(s)$ and therefore of $\Lambda_{\mathrm{ex}}(s)$ is zero. For this value of $\phi, \Lambda_{\text {ex }}$ coincides with $\Lambda_{\text {static }}$ defined in (72) and $N_{\phi}$ is equal to $N_{0}$ of equation (77).

[1] M. J. Steel, M. K. Olsen, L. I. Plimak, P. D. Drummond, S. M. Tan, M. J. Collett, D. F. Walls, and R. Graham, Phys. Rev. A 58, 4824 (1998).

[2] D. F. Walls and G. J. Milburn, Quantum Optics, Springer-Verlag, Berlin (1994).

[3] A. Sinatra, C. Lobo and Y. Castin, Phys. Rev. Lett. 87, 210404 (2001).

[4] A. Sinatra, Y. Castin and C. Lobo, Jour. of Mod. Opt. 47, 2629-2644 (2000).

[5] Y. Castin and R. Dum, Phys. Rev. A 57 3008-3021 (1998).

[6] C. Gardiner, Phys. Rev. A 56 1414-1423 (1997).

[7] About the solution of the exact problem see e.g. I. Carusotto, Y. Castin, and J. Dalibard, Phys. Rev. A 63, 023606 (2001) and L. I. Plimak, M. K. Olsen, M. Fleischhauer and M. J. Collett, Europhys. Lett. 56, 372-378 (2001).

[8] Actually the equation for $\psi$ obtained in the truncated Wigner approach differs from the usual Gross-Pitaevskii equation by a term involving a sum over all modes $\phi_{k}(r)$ of the field. In this paper we restrict ourselves to the case where $\sum_{k} \phi_{k}(r) \phi_{k}^{*}(r)$ is a constant independent of the position $r$. In this case the mean field term in the truncated Wigner point of view differs from the one $g|\psi|^{2}$ of the usual Gross-Pitaevskii equation by a constant term involving the number of modes, see [1] and our section [1] below.

[9] Yu. Kagan and B. Svistunov, Phys. Rev. Lett. 793331 (1997) and references therein.

[10] K. Damle, S. Majumdar, and S. Sachdev, Phys. Rev. A 54, 5037 (1996).

[11] M.J. Davis, S.A. Morgan and K. Burnett, Phys. Rev. Lett. 87, 160402 (2001).

[12] K. Góral, M. Gajda, K. Rzążewski, Opt. Express 8, 82 (2001).

[13] M.J. Davis, S.A. Morgan and K. Burnett, cond-mat/0201571.

[14] C. Gardiner, Quantum Noise, Springer-Verlag Berlin Heidelberg (1991), chapter 4 "Phase space methods".

[15] In the case of a harmonically trapped gas we assume that the box is large enough so that the atomic density is small close to the boundaries.

[16] Y. Castin, lectures in: Coherent Atomic Matter Waves, Les Houches Summer School Session LXXII in 1999, edited by R. Kaiser, C. Westbrook, and F. David (Springer, New York, (2001), and cond-mat/0105058.

[17] William H. Press, Brian P. Flannery, Saul A. Teukolsky, William T. Vetterling, Numerical Recipes, §7.2, Cambridge University Press (1986).

[18] M. Wilkens, C. Weiss, Opt. Expr 1, 272 (1997).

[19] S. Giorgini, L. P. Pitaevskii, S. Stringari, Phys. Rev. Lett. 80, 5040, (1998).

[20] V.V. Kocharovsky, Vl. V. Kocharovsky, and Marlan O. Scully, Phys. Rev. Lett. 84, 2306 (2000).

[21] We have included not only the leading terms but also higher order terms in the denominator of the $3 \mathrm{D}$ formula since we have observed numerically that this dramatically improves the accuracy of the formula for moderately high values of $k_{B} T / \hbar \omega$.

[22] see I. Carusotto and Y. Castin (in preparation).

[23] exact in the sense of O. Penrose and L. Onsager, Phys. Rev. 104, 576 (1956).

[24] We find at last the physical interpretation of a component of $\phi^{(2)}$ that was unexplained in [5].

[25] Y. Castin and R. Dum, Phys. Rev. Lett. 79, 3553 (1997).

[26] D. Stamper-Kurn, A. Chikkatur, A. Görlitz, S. Inouye, S. Gupta, D. Pritchard, W. Ketterle, Phys. Rev. Lett. 82, 4569 (1999).

[27] W. Ketterle, Spinor Condensates and Light Scattering from Bose-Einstein Condensates, in Les Houches Summer School 1999, Session LXXII, eds. R. Kaiser and C. Westbrook (cond-mat/0005001)

[28] In the simulation we observe damping in each single realisation, which indicates that this damping is a true relaxation phenomenon and not a collapse due to dephasing among different stochastic realisations. This was not the case in the 1D model of [3].

[29] We note that exactly the same procedure from the beginning of this subsection can be followed in the quantum treatment. The evolution equation for $\hat{\Lambda}_{\text {ex }}$ can in fact be obtained from (F2) by putting hats on $a_{\phi}$ and $\psi_{\perp}$ and by changing stars 
into daggers (see equation (A3) of []ㅡ)

[30] For the parameters of figure (3) we perform a linear fit of the perturbative prediction for $\left|\left\langle b_{0}(t)\right\rangle\right|$ on the time interval $(0.0075,0.015)$ in units of $m L^{2} / \hbar$ for a grid size $48^{3}$. The slope is -0.328 with a linear correlation coefficient -0.99998 . The results are the same for a grid size $64^{3}$.

[31] In the perturbative calculation for the figure we have actually included the effect of the interaction between Bogoliubov modes which provides damping also in the excitation phase, simply by adding a term $-i \hbar \gamma_{\text {perturb }} b_{0}$ and $-i \hbar \gamma_{\text {perturb }} b_{-0}$ to the right hand side of (79) and of (80) respectively. This damping term is not totally negligible indeed since $\gamma_{\text {perturb }} t_{\text {exc }} \simeq$ 0.1 .

[32] Vincent Liu, Phys. Rev. Lett. 79, 4056 (1997).

[33] L. P. Pitaevskii, and S. Stringari, Phys. Lett. A 235, 398 (1997).

[34] P. O. Fedichev and G. V. Shlyapnikov, Phys. Rev. A 58, 3146 (1998).

[35] We have checked with the particular example $\mathcal{N}=22^{3}, N=5 \times 10^{4}, k_{B} T=3 \mu$ and $\mu=500 \hbar^{2} / \mathrm{mL}^{2}$, that the mean Bogoliubov energy (after averaging over 100 realisations) is conserved during the course of the time evolution at the $2 \%$ level.

[36] Michele Modugno, Ludovic Pricoupenko, and Yvan Castin, cond-mat/0203597.

[37] We have used the inequality $x / \tanh x<1+x^{2} / 3$.

[38] H. T. C. Stoof, J. Low Temp. Phys. 114, 1 (1999).

[39] C. W. Gardiner, J. R. Anglin, and T. I. A. Fudge, cond-mat/0112129.

[40] Ibidem [17], §5.6. 